\title{
One-dimensional arrangements of metal atoms in transition metal carbonyl complexes of mixed main group metal alkoxides
}

\author{
Michael Veith, Stefan Weidner, Klaus Kunze, Dieter Käfer, Johannes Hans and \\ Volker Huch \\ Institute for Inorganic Chemistry, University of the Saarland, Postfach 1511 50, D-66041 Saarbrücken \\ (Germany)
}

(Received 30 October 1993; accepted 15 December 1993)

\section{CONTENTS}

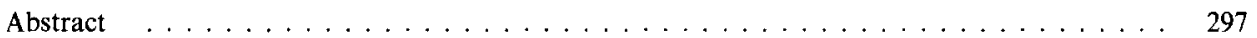

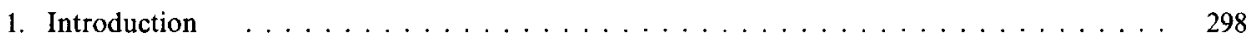

2. Syntheses of mixed metal alkoxide carbonyl complexes . . . . . . . . . . . . . . . . 298

2.1. Mixed main group metal alkoxides with $n s^{2}$-configurated elements . . . . . . . . 298

2.2. Synthesis of transition metal carbonyl complexes $\ldots \ldots \ldots \ldots$. . . . . . . . . . . . . .

2.3. Reactivity of $n \mathbf{s}^{2}$-configurated main group elements towards transition metals . . . 302

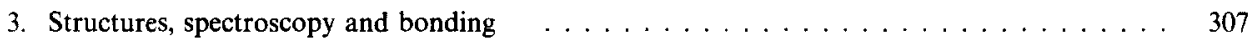

3.1. Structural characterization of $n \mathrm{~s}^{2}$-configurated main group metal alkoxide transition metal carbonyl complexes $\ldots \ldots \ldots \ldots \ldots 7 \ldots \ldots \ldots$

3.2. IR frequencies of $\mathrm{CO}$ in metal carbonyl complexes of mixed metal alkoxides . . . . 314

3.3. Some comments on a cluster concept $\ldots \ldots \ldots \ldots \ldots \ldots \ldots$

4. Oligomers and polymers with one-dimensionally arranged metal atoms . . . . . . . 318

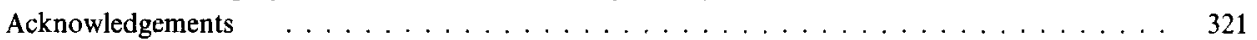

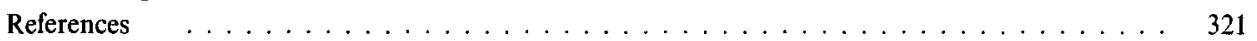

\begin{abstract}
Mixed metal alkoxides containing main group elements with $n \mathrm{~s}^{2}$ electron configurations of the general formula $\mathrm{M}\left(\mathrm{O}^{\mathrm{t}} \mathrm{Bu}\right)_{3} \mathrm{M}^{\prime}\left(\mathrm{M}=\mathrm{In}, \mathrm{Tl} ; \mathrm{M}^{\prime}=\mathrm{Ge}, \mathrm{Sn}, \mathrm{Pb}\right), \mathrm{M}^{\prime}\left(\mathrm{O}^{t} \mathrm{Bu}\right)_{3} \mathrm{M}\left(\mathrm{O}^{\mathrm{t}} \mathrm{Bu}\right)_{3} \mathbf{M}^{\prime}(\mathrm{M}=\operatorname{divalent}$ metal atom, $\left.\mathbf{M}^{\prime}=\mathrm{Ge}, \mathrm{Sn}\right)$ and $\left(\mathrm{O}^{t} \mathrm{Bu}\right) \mathrm{M}^{\prime}\left(\mathrm{O}^{t} \mathrm{Bu}\right)_{2} \mathrm{M}\left(\mathrm{O}^{t} \mathrm{Bu}\right)_{2} \mathrm{M}\left(\mathrm{O}^{t} \mathrm{Bu}\right)_{2} \mathrm{M}^{\prime}\left(\mathrm{O}^{t} \mathrm{Bu}\right)\left(\mathrm{M}=\mathrm{Co}, \mathrm{Ni}^{\prime} \mathrm{M}^{\prime}=\mathrm{Ge}, \mathrm{Sn}\right)$ can be reacted with simple metal carbonyls. The products obtained are composed of a one-dimensional array of metal atoms held together by direct metal-metal bonds or by bridging alkoxide groups. The following compounds have been isolated and characterized by IR and NMR spectroscopy and X-ray diffraction $\left(\mathrm{M}^{\prime}=\mathrm{Ge}, \mathrm{Sn}, \mathrm{Pb} ; \mathrm{M}=\mathrm{In}, \mathrm{Tl} ; \mathrm{M}^{\mathrm{T}}=\right.$ transition metal): $\mathbf{M}^{\prime}\left(\mathrm{O}^{\mathrm{t}} \mathrm{Bu}\right)_{3} \mathrm{M}-\mathrm{M}^{\mathrm{T}}(\mathrm{CO})_{n}, \quad(\mathrm{CO})_{n} \mathrm{M}^{\mathrm{T}}-$ $\mathrm{M}\left(\mathrm{O}^{\mathrm{t} B u}\right)_{3} \mathrm{M}^{\prime}-\mathrm{M}^{\mathrm{T}}(\mathrm{CO})_{n}, \quad \mathrm{M}^{\prime}\left(\mathrm{O}^{\mathrm{t}} \mathrm{Bu}\right)_{3} \mathrm{M}-\mathrm{M}^{\mathrm{T}}(\mathrm{CO})_{n} \mathrm{M}\left(\mathrm{O}^{t} \mathrm{Bu}\right)_{3} \mathrm{M}^{\prime}, \quad(\mathrm{CO})_{n} \mathrm{M}^{\mathrm{T}}-\mathrm{M}^{\prime}\left(\mathrm{O}^{\mathrm{t}} \mathrm{Bu}\right)_{3} \mathrm{M}\left(\mathrm{O}^{\mathrm{t}} \mathrm{Bu}\right)_{3} \mathrm{M}^{\prime}-$ $\mathrm{M}^{\mathrm{T}}(\mathrm{CO})_{n}, \quad(\mathrm{CO})_{n} \mathrm{M}^{\mathrm{T}}-\mathrm{M}^{\prime}\left[\mathrm{O}^{t} \mathrm{Bu}\right]\left(\mathrm{O}^{t} \mathrm{Bu}\right)_{2} \mathrm{M}\left(\mathrm{O}^{\mathrm{t}} \mathrm{Bu}\right)_{2} \mathrm{M}\left(\mathrm{O}^{\mathrm{t} B u}\right)_{2}\left[\mathrm{O}^{\mathrm{t}} \mathrm{Bu}\right] \mathrm{M}^{\prime}-\mathrm{M}^{\mathrm{T}}(\mathrm{CO})_{n} \quad$ and $\quad(\mathrm{CO})_{n} \mathrm{M}^{\mathrm{T}}-$ $\mathrm{M}^{\prime}\left(\mathrm{O}^{\mathrm{t}} \mathrm{Bu}\right)_{3} \mathrm{M}-\mathrm{M}^{\mathrm{T}}(\mathrm{CO})_{n} \mathrm{M}\left(\mathrm{O}^{\mathrm{t}} \mathrm{Bu}\right)_{3} \mathrm{M}^{\prime}-\mathrm{M}^{\mathrm{T}}(\mathrm{CO})_{n}$. In addition, an oligomeric species of the general formula $\left[\mathrm{Sn}\left(\mathrm{O}^{t} \mathrm{Bu}\right)_{3} \mathrm{In}-\mathrm{Mo}(\mathrm{CO})_{4}\right]_{n}-\mathrm{Sn}\left(\mathrm{O}^{t} \mathrm{Bu}\right)_{3} \mathrm{In}-\mathrm{Mo}(\mathrm{CO})_{5}$ with $n \approx 9,10$ has been isolated.
\end{abstract}

Correspondence to: M. Veith, Institute for Inorganic Chemistry, University of the Saarland, Postfach 1511 50, D-66041 Saarbrücken, Germany. 
1. INTRODUCTION

It is well known that the transition metals may be coordinated by a wide variety of different ligands, most of which acting generally as electron donors. Classical $\sigma$-donor ligands such as $\mathrm{NH}_{3}$ and $\mathrm{OH}_{2}$ or $\sigma$-donors and $\pi$-acceptors such as $\mathrm{CO}$ and $\mathrm{PR}_{\mathbf{3}}$ contain non-metallic elements which possess electron lone pairs and act as coordination centres to the transition metals. Another large group of ligands can be classified as $\pi$-systems, in which various atoms take part in bonding to the transition metal at the same time through the $\pi$ ligand orbitals ( $\pi$-complex). In addition to these classical ligands there is a comparatively small number of systems known in which a central metallic element acts as an electron donor. Most of these metals are main group elements in a low oxidation state, in particular the $n \mathrm{~s}^{2}$ electron configuration, e.g. $\mathrm{Ge}(\mathrm{II})$ and $\mathrm{Sn}(\mathrm{II})$ [1].

Some years ago we reported the synthesis of metal alkoxides which combine one or two main group metals in the $n s^{2}$ configuration [2-4]. Compounds of type $\quad \mathrm{M}\left(\mathrm{O}^{t} \mathrm{Bu}\right)_{3} \mathrm{M}^{\prime} \quad\left[\mathrm{M}=\mathrm{In}(\mathrm{I}), \quad \mathrm{Tl}(\mathrm{I}), \quad \mathrm{M}^{\prime}=\mathrm{Ge}(\mathrm{II}), \quad \mathrm{Sn}(\mathrm{II}), \quad \mathrm{Pb}(\mathrm{II})\right] \quad$ and $\mathrm{M}^{\prime}\left(\mathrm{O}^{t} \mathrm{Bu}\right)_{3} \mathrm{M}\left(\mathrm{O}^{t} \mathrm{Bu}\right)_{3} \mathrm{M}^{\prime} \quad\left[\mathrm{M}=\right.$ divalent metal cation, $\left.\mathrm{M}^{\prime}=\mathrm{Ge}(\mathrm{II}), \mathrm{Sn}(\mathrm{II}), \mathrm{Pb}(\mathrm{II})\right]$ are of special interest, as they display two low-valent metallic elements which are situated on the axial sites of one or two (fused) trigonal bipyramids, thus making them accessible for complexation with transition metal carbonyls. This synthetic procedure should allow the construction of molecules with one-dimensionally arranged metallic elements. As will be discussed below, this can indeed be accomplished, and we have denoted these alkoxide compounds in view of their special structure and reactivity as "Janus-Head" molecules [5].

This review summarizes the mixed metal alkoxide-transition metal carbonyl complexes synthesized so far in our laboratory. Special consideration will be given to the structural properties of these compounds. A comparative study provides insight into the reactivity of low-valent elements in the $n \mathrm{~s}^{2}$ electron configuration.

2. SYNTHESIS OF MIXED METAL ALKOXIDE CARBONYL COMPLEXES

\subsection{Mixed metal alkoxides with $n s^{2}$-configurated elements}

Mixed metal alkoxides of different metallic elements can be prepared by well established routes [6-8]. For alkoxides containing elements in the $n \mathrm{~s}^{2}$ configuration, and with tert-butyl as the organic group, the following procedures [eqns. (1)-(3)] have been found to provide good yields.

$$
\begin{aligned}
& \mathrm{M}\left(\mathrm{O}^{\mathrm{t} B u}\right)_{n}+n\left({ }^{\mathrm{t}} \mathrm{BuO}\right)_{2} \mathrm{M}^{\prime} \rightarrow \mathrm{M}_{n}^{\prime} \mathrm{M}\left(\mathrm{O}^{\mathrm{t}} \mathrm{Bu}\right)_{(2 n+n)} \\
& \mathrm{M}=\mathrm{Tl}(\mathrm{I}) ; n=1 ; \mathrm{M}^{\prime}=\mathrm{Ge}(\mathrm{II}), \mathrm{Sn}(\mathrm{II})
\end{aligned}
$$


$\mathrm{M}=\mathrm{Ca}, \mathrm{Sr}, \mathrm{Ba}, \mathrm{Pb}, \mathrm{Eu} ; n=2, \mathrm{M}^{\prime}=\mathrm{Ge}(\mathrm{II}), \mathrm{Sn}(\mathrm{II}), \mathrm{Pb}(\mathrm{II})$

$\mathrm{MX}_{n}+n \mathrm{Na}\left({ }^{\mathrm{t}} \mathrm{BuO}\right)_{3} \mathrm{M}^{\prime} \rightarrow n \mathrm{NaX}+\mathrm{M}\left[\left(\mathrm{O}^{\mathrm{t}} \mathrm{Bu}\right)_{3} \mathrm{M}^{\prime \prime}\right]_{n}$

$\mathrm{M}=\mathrm{In}(\mathrm{I}), \mathrm{Tl}(\mathrm{I}) ; n=1 ; \mathrm{M}^{\prime}=\mathrm{Ge}(\mathrm{II}), \mathrm{Sn}(\mathrm{II}), \mathrm{Pb}(\mathrm{II})$

$\mathrm{M}=(\mathrm{Mg}), \mathrm{Ca}, \mathrm{Sr}, \mathrm{Ba}, \mathrm{Cd}, \mathrm{Eu} ; n=2 ; \mathrm{M}^{\prime}=\mathrm{Ge}(\mathrm{II}), \mathrm{Sn}(\mathrm{II}), \mathrm{Pb}(\mathrm{II})$

$2(\mathrm{D}) \mathrm{M}^{\prime} \mathrm{X}_{2}+3 \mathrm{M}\left(\mathrm{O}^{\mathrm{t} B u}\right)_{2} \rightarrow 2 \mathrm{MX}_{2}+2 \mathrm{D}+\mathrm{M}^{\prime}\left(\mathrm{O}^{\mathrm{t} B u}\right)_{3} \mathrm{M}\left(\mathrm{O}^{\mathrm{t}} \mathrm{Bu}\right)_{3} \mathrm{M}^{\prime}$

$\mathrm{M}=\mathrm{Mg}, \mathrm{Ca} ; \mathrm{M}^{\prime}=\mathrm{Ge}(\mathrm{II})(\mathrm{D}=$ dioxane), $\mathrm{Sn}(\mathrm{II}), \mathrm{Pb}(\mathrm{II})$

All reactions are performed in non-coordinating solvents and the yields of the products are $70-95 \%$. The products are purified by sublimation or crystallization; however the choice of the reaction procedure may influence the yield [9]. In the $\mathrm{M}\left(\mathrm{O}^{\mathrm{t}} \mathrm{Bu}\right)_{3} \mathrm{M}^{\prime}$ series, the synthesis of $\mathrm{Tl}\left(\mathrm{O}^{t} \mathrm{Bu}\right)_{3} \mathrm{Sn}[10]$ and $\mathrm{In}\left(\mathrm{O}^{\mathrm{t} B u}\right)_{3} \mathrm{Sn}$ [11] have been described earlier. By similar routes, $\mathrm{Tl}\left(\mathrm{O}^{t} \mathrm{Bu}\right)_{3} \mathrm{Ge}, \mathrm{Tl}\left(\mathrm{O}^{\mathrm{t}} \mathrm{Bu}\right)_{3} \mathrm{~Pb}$ and $\operatorname{In}\left(\mathrm{O}^{\prime} \mathrm{Bu}\right)_{3} \mathrm{Ge}$ have been obtained and characterized completely, and selected data are given in Table 1 . As an alternative to $\left[\mathrm{Na}\left(\mathrm{O}^{t} \mathrm{Bu}\right)_{3} \mathrm{M}^{\prime}\right]_{2}[12], \mathrm{Tl}\left(\mathrm{O}^{\mathrm{t}} \mathrm{Bu}\right)_{3} \mathrm{M}^{\prime}$ may be used by reaction with $\mathrm{InBr}$, as shown in eqn. (4).

$\mathrm{Tl}\left(\mathrm{O}^{t} \mathrm{Bu}\right) \mathrm{M}^{\prime}+\mathrm{InBr} \rightarrow \mathrm{TlBr}+\mathrm{In}\left(\mathrm{O}^{t} \mathrm{Bu}\right)_{3} \mathrm{M}^{\prime}$

$\mathrm{M}^{\prime}=\mathrm{Ge}(\mathrm{II}), \mathrm{Sn}(\mathrm{II})$

So far we have been unsuccessful in synthesizing $\operatorname{In}\left(\mathrm{O}^{t} \mathrm{Bu}\right)_{3} \mathrm{~Pb}$ by any of the routes described above.

Transition metals with a $3 \mathrm{~d}$ configuration can be combined with $\mathrm{Ge}$ (II), $\mathrm{Sn}$ (II) and $\mathrm{Pb}(\mathrm{II}$ ) using tert-butoxy groups as bridging ligands. If sodium tris(tert-butoyxy)germate or -plumbate is allowed to react with chlorides of $\mathrm{Cr}, \mathrm{Mn}$ or $\mathrm{Zn}$, compounds of the type $\mathrm{M}^{\prime}\left(\mathrm{O}^{\prime} \mathrm{Bu}\right)_{3} \mathrm{M}\left(\mathrm{O}^{t} \mathrm{Bu}\right)_{3} \mathrm{M}^{\prime}$ are formed [eqn. (5)] [13].

$\left[\mathrm{Na}\left(\mathrm{O}^{\mathrm{t}} \mathrm{Bu}\right)_{3} \mathrm{M}^{\prime}\right]_{2}+\mathrm{MCl}_{2} \rightarrow \mathrm{M}^{\prime}\left(\mathrm{O}^{t} \mathrm{Bu}\right)_{3} \mathrm{M}\left(\mathrm{O}^{\mathrm{t}} \mathrm{Bu}\right)_{3} \mathrm{M}^{\prime}+2 \mathrm{NaCl}$

$\mathrm{M}=\mathrm{Cr}, \mathrm{Mn},(\mathrm{Zn}) ; \mathrm{M}^{\prime}=\mathrm{Ge}, \mathrm{Pb}$

On the other hand, if sodium tris(tert-butoxy)stannate, -germate or -plumbate is reacted with the chlorides of the smaller sized $3 \mathrm{~d}$ elements, a new type of compound can be isolated from the reaction mixture, which has the general formula $\left({ }^{t} \mathrm{BuO}\right) \mathrm{M}^{\prime}\left(\mathrm{O}^{\mathrm{t}} \mathrm{Bu}\right)_{2} \mathrm{M}\left(\mathrm{O}^{\mathrm{t}} \mathrm{Bu}\right)_{2} \mathrm{M}\left(\mathrm{O}^{t} \mathrm{Bu}\right)_{2} \mathrm{M}^{\prime}\left(\mathrm{O}^{t} \mathrm{Bu}\right)$ and which combines two main group and two transition metals [eqn. (6)] [13].

$4\left[\mathrm{Na}\left(\mathrm{O}^{\mathrm{t} B u}\right)_{3} \mathrm{M}^{\prime}\right]_{2}+6 \mathrm{MX}_{2} \rightarrow$

$3\left({ }^{t} \mathrm{BuO}\right) \mathrm{M}^{\prime}\left(\mathrm{O}^{\mathrm{t}} \mathrm{Bu}\right)_{2} \mathrm{M}\left(\mathrm{O}^{\mathrm{t}} \mathrm{Bu}\right)_{2} \mathrm{M}\left(\mathrm{O}^{\mathrm{t}} \mathrm{Bu}\right)_{2} \mathrm{M}^{\prime}\left(\mathrm{O}^{\mathrm{t}} \mathrm{Bu}\right)+8 \mathrm{NaX}+2 \mathrm{M}^{\prime} \mathrm{X}_{2}$

$\mathrm{M}^{\prime}=\mathrm{Ge} ; \mathrm{M}=\mathrm{Co}, \mathrm{Ni} ; \mathrm{X}=\mathrm{Cl}, \mathrm{Br}$

$\mathrm{M}^{\prime}=\mathrm{Sn} ; \mathrm{M}=\mathrm{Cr}, \mathrm{Mn}, \mathrm{Co}, \mathrm{Ni} ; \mathrm{X}=\mathrm{Cl}, \mathrm{Br}, \mathrm{I}$ 
TABLE 1

Some data for compounds of the general formula $\mathrm{M}\left(\mathrm{O}^{t} \mathrm{Bu}\right)_{3} \mathrm{M}^{\prime}$

\begin{tabular}{|c|c|c|c|c|}
\hline Compound & $\begin{array}{l}\text { Space group } \\
\text { and lattice } \\
\text { constants }\end{array}$ & $\begin{array}{l}{ }^{1} \mathrm{H} \text { NMR } \\
(\delta \mathrm{ppm})\end{array}$ & $\begin{array}{l}\text { Melting } \\
\text { point } /{ }^{\circ} \mathrm{C}\end{array}$ & Synthesis \\
\hline $\operatorname{In}\left(\mathrm{O}^{\mathrm{t}} \mathrm{Bu}\right)_{3} \mathrm{Ge}^{\mathrm{a}}$ & $\begin{array}{l}\text { Pnma } \\
a=12.53(1) \\
b=15.51(2) \\
c=9.604(8) \\
Z=4\end{array}$ & 1.39 & $52-54$ & $\begin{array}{l}\text { (a) } \mathrm{Tl}\left(\mathrm{O}^{\mathrm{t}} \mathrm{Bu}\right)_{3} \mathrm{Ge}+\mathrm{InBr} \text {, } \\
\text { yield } 77.4 \% \\
\text { (b) }\left[\mathrm{Na}\left(\mathrm{O}^{t} \mathrm{Bu}\right)_{3} \mathrm{Ge}\right]_{2}+\mathrm{InBr} \text {, } \\
\text { yield } 71.8 \%\end{array}$ \\
\hline $\operatorname{In}\left(\mathrm{O}^{t} \mathrm{Bu}\right)_{3} \mathrm{Sn}[10]$ & $\begin{array}{l}P 6_{3} / m \\
a=b=9.867(9) \\
c=11.21(1) \\
Z=2\end{array}$ & 1.37 & 41 & $\begin{array}{l}\text { (a) } \mathrm{Tl}\left(\mathrm{O}^{t} \mathrm{Bu}\right)_{3} \mathrm{Sn}+\mathrm{InBr} \text {, } \\
\text { yield } 92 \% \\
\text { (b) }\left[\mathrm{Na}\left(\mathrm{O}^{t} \mathrm{Bu}\right)_{3} \mathrm{Sn}\right]_{2}+\mathrm{InBr} \text {, } \\
\text { yield } 90 \%\end{array}$ \\
\hline $\mathrm{Tl}\left(\mathrm{O}^{\mathrm{t}} \mathrm{Bu}\right)_{3} \mathrm{Ge}^{\mathrm{a}}$ & $\begin{array}{l}\text { Pnma } \\
a=12.361(9) \\
b=15.64(1) \\
c=9.604(7) \\
Z=4\end{array}$ & 1.38 & $87-88$ & $\begin{array}{l}{\left[\mathrm{Tl}\left(\mathrm{O}^{t} \mathrm{Bu}\right)\right]_{4}+\left[\mathrm{Ge}\left(\mathrm{O}^{t} \mathrm{Bu}\right)_{2}\right]_{2},} \\
\quad \text { yield } 92.9 \%\end{array}$ \\
\hline $\mathrm{Tl}\left(\mathrm{O}^{t} \mathrm{Bu}\right)_{3} \mathrm{Sn}[9]$ & $\begin{array}{l}P 6_{3} / m \\
a=b=9.944(5) \\
c=11.07(1) \\
Z=2\end{array}$ & 1.32 & 43 & $\begin{array}{l}{\left[\mathrm{Tl}\left(\mathrm{O}^{\mathrm{t} B u}\right)\right]_{4}} \\
\quad \text { yield } 90 \%\end{array}$ \\
\hline $\mathrm{Tl}\left(\mathrm{O}^{\mathrm{t}} \mathrm{Bu}\right)_{3} \mathrm{~Pb}^{\mathrm{a}}$ & $\begin{array}{l}P 6_{3} / m \\
a=b=9.94(5) \\
c=10.80(9) \\
Z=2\end{array}$ & 1.31 & $73-74$ & $\begin{array}{l}{\left[\mathrm{Tl}\left(\mathrm{O}^{t} \mathrm{Bu}\right)\right]_{4}+\left[\mathrm{Pb}\left(\mathrm{O}^{t} \mathrm{Bu}\right)_{2}\right]_{3},} \\
\quad \text { yield } 71.7 \%\end{array}$ \\
\hline
\end{tabular}

${ }^{a}$ Analytical data: for $\operatorname{In}\left(\mathrm{O}^{t} \mathrm{Bu}\right)_{3} \mathrm{Ge}$, calc. C 35.44, H 6.69, found C 35.35 , H 6.72\%; for $\mathrm{Tl}\left(\mathrm{O}^{t} \mathrm{Bu}\right)_{3} \mathrm{Ge}$, calc. $\mathrm{C} 29.04, \mathrm{H} 5.48$, found $\mathrm{C} 29.11, \mathrm{H} 5.58 \%$; and for $\mathrm{Tl}\left(\mathrm{O}^{t} \mathrm{Bu}\right)_{3} \mathrm{~Pb}$, calc. C $22.85, \mathrm{H} 4.31$, found C $22.70, \mathrm{H} 4.34 \%$.

$\mathrm{M}^{\prime}=\mathrm{Pb} ; \mathrm{M}=\mathrm{Co} ; \mathrm{X}=\mathrm{Cl}$

In these compounds the transition metal is in a fourfold coordination site which can be described as a spiro metallic centre in a distorted oxygen tetrahedron [13].

An example of each of the different mixed metal alkoxides that have been used in the preparation of metal carbonyl complexes is shown in Fig. 1. The $\mathrm{M}\left(\mathrm{O}^{t} \mathrm{Bu}\right)_{3} \mathrm{M}^{\prime}$ structure is the simplest, with a central $\mathrm{MO}_{3} \mathbf{M}^{\prime}$ core that can be described as a distorted trigonal bipyramid with the three oxygen atoms assembled in the equatorial plane and the two metallic elements in the apical positions. $\mathrm{M}^{\prime}\left(\mathrm{O}^{t} \mathrm{Bu}\right)_{3} \mathrm{M}\left(\mathrm{O}^{t} \mathrm{Bu}\right)_{3} \mathrm{M}^{\prime}$ can be formally viewed as two trigonal bipyramids fused together at the common divalent metallic element, which can either be a main group, a transition or a lanthanide (e.g. Eu) element. The $\left({ }^{t} \mathrm{BuO}\right) \mathrm{M}^{\prime}\left(\mathrm{O}^{t} \mathrm{Bu}\right)_{2} \mathrm{M}\left(\mathrm{O}^{t} \mathrm{Bu}\right)_{2} \mathrm{M}\left(\mathrm{O}^{\mathrm{t}} \mathrm{Bu}\right)_{2} \mathrm{M}^{\prime}\left(\mathrm{O}^{\mathrm{t}} \mathrm{Bu}\right)$ compounds have four metallic elements which are linked together by ${ }^{\mathrm{t}} \mathrm{BuO}$ bridges, 


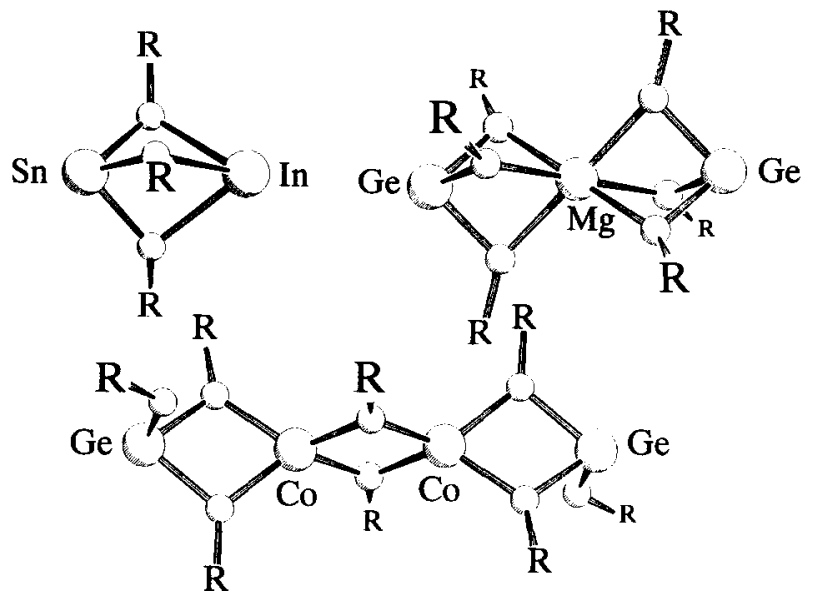

Fig. 1. Skeleton structure of three different mixed metal alkoxides: $\mathrm{Sn}(\mathrm{OtBu})_{3}$ In [11], $\mathrm{Ge}(\mathrm{OtBu})_{3} \mathrm{Mg}(\mathrm{OtBu})_{3} \mathrm{Ge}[9]$ and $(\mathrm{BuO}) \mathrm{Ge}(\mathrm{OtBu})_{2} \mathrm{Co}(\mathrm{OtBu})_{2} \mathrm{Co}(\mathrm{OtBu})_{2} \mathrm{Ge}(\mathrm{OtBu})[13](\mathrm{R}=$ tert-Butyl; the non-designated balls represent oxygen atoms).

and three four-membered rings are arranged in a perpendicular fashion. The atoms $\mathrm{M}$ are in a distorted tetrahedral environment whereas the $\mathbf{M}^{\prime}$ atoms are in a trigonal pyramidal coordination. Each end of the compound $\left(\mathrm{M}^{\prime}\right)$ is terminated by a tertbutoxy group and the oxygen atom is only dicoordinate.

\subsection{Syntheses of transition metal carbonyl complexes}

The formation of an $\mathbf{M}^{\mathrm{T}}-\mathbf{M}^{\prime}$ bond $\left(\mathrm{M}^{\mathrm{T}}=\right.$ transition metal, $\mathbf{M}^{\prime}=n \mathrm{~s}^{2}$ element) can be accomplished by two different procedures which are outlined in eqns. (7) and (8).

$\mathrm{X}(\mathrm{RO})_{3} \mathrm{M}^{\prime}+(\mathrm{CO})_{n} \mathrm{M}^{\mathrm{T}} \rightarrow \mathrm{X}(\mathrm{RO})_{3} \mathrm{M}^{\prime}-\mathrm{M}^{\mathrm{T}}(\mathrm{CO})_{n-1}+\mathrm{CO}$

$\mathrm{M}^{\mathrm{T}}(\mathrm{CO})_{n} \underset{\mathrm{THF}}{\stackrel{h v}{\longrightarrow}} \mathrm{M}^{\mathrm{T}}(\mathrm{CO})_{n-1}(\mathrm{THF})+\mathrm{CO}$

$$
\mathrm{M}^{\mathrm{T}}(\mathrm{CO})_{n-1}(\mathrm{THF})+\mathrm{M}^{\prime}(\mathrm{OR})_{3} \mathrm{X} \rightarrow(\mathrm{CO})_{n-1} \mathrm{M}^{\mathrm{T}}-\mathrm{M}^{\prime}(\mathrm{OR})_{3} \mathrm{X}+\mathrm{THF}
$$

$\mathrm{X}=\mathrm{M}^{\prime}$ or $\mathrm{M}(\mathrm{OR})_{3} \mathrm{M}^{\prime}$

Reaction (7) may be described as a thermally induced $\mathrm{CO}$ substitution by a metal base. In contrast, reaction (8) relies on the photochemical formation of a labile THF complex which undergoes subsequent thermal reaction to lose the THF ligand, allowing for complexation of the $\boldsymbol{n s}^{2}$-metal centre. These well known reactions have been widely used in transition metal chemistry $[14,15]$ have have been transferred to alkoxides of simple $n s^{2}$ elements some time ago [16-18]. When two ligand sites at a transition metal have to be displaced simultaneously, the norbornadiene (nbd) 
route has proved very successful [19] and has been adopted for $n s^{2}$-metal alkoxides [eqn. (9)].

$$
\begin{aligned}
& \mathrm{M}(\mathrm{CO})_{n}+\mathrm{nbd} \rightarrow \mathrm{M}(\mathrm{CO})_{n-2} \mathrm{nbd}+2 \mathrm{CO} \\
& \mathrm{M}(\mathrm{CO})_{n-2} \mathrm{nbd}+2 \mathrm{M}^{\prime}(\mathrm{OR})_{3} \mathrm{X} \rightarrow \mathrm{X}(\mathrm{OR})_{3} \mathrm{M}^{\prime}-\mathrm{M}(\mathrm{CO})_{n-2}-\mathrm{M}^{\prime}(\mathrm{OR})_{3} \mathrm{X}+\mathrm{nbd}
\end{aligned}
$$

Table 2 lists the mixed metal alkoxide transition metal carbonyl complexes, together with the synthetic procedure used and the experimental conditions. The isolated yields of the products can attain $95 \%$. The compounds are abbreviated with the first letters of the metallic elements. Thus TSM designates the compound $\mathrm{Tl}\left(\mathrm{O}^{\mathrm{t}} \mathrm{Bu}\right)_{3} \mathrm{SnMo}(\mathrm{CO})_{5}$ and $\mathrm{CSESC}$ the compound $(\mathrm{CO})_{5} \mathrm{CrSn}(\mathrm{OtBu})_{3} \mathrm{Eu}(\mathrm{OtBu})_{3}$ $\mathrm{SnCr}(\mathrm{CO})_{5}$; the number of letters is equal to the number of metallic elements present in the compound. In some instances different elements are designated by the same letter (e.g. Sn and Sr), but it is clear from the order of the letters (which is strictly structural) that FSSSF denotes the compound $(\mathrm{CO})_{4} \mathrm{Fe}-\mathrm{Sn}\left(\mathrm{O}^{t} \mathrm{Bu}\right)_{3} \mathrm{Sr}\left(\mathrm{O}^{t} \mathrm{Bu}\right)_{3} \mathrm{SnFe}(\mathrm{CO})_{4}$ and the middle $\mathrm{S}$ refers to strontium (with respect to the alkoxides used another interpretation would not make sense).

The easiest bonding formation $\mathrm{M}^{\prime}-\mathrm{M}^{\mathrm{T}}\left(\mathrm{M}^{\prime}=\right.$ metal with $n \mathrm{~s}^{2}$ configuration, $\mathrm{M}^{\mathrm{T}}=$ transition metal) is accomplished using the method depicted in eqn. (8), which can be performed under mild conditions (see Table 2). The thermal carbonyl displacement method [eqn. (7)] may require high temperatures and long reaction times when carbonyls of $\mathrm{Cr}, \mathrm{Mo}$ or $\mathrm{W}$ are used. The norbornadiene displacement [eqn. (9)] requires higher temperatures and longer heating than the THF displacement. This difference can be attributed to the fact that the norbornadiene is more strongly bound than THF to the transition metal [19].

As indicated in Table 2, the products of reactions (7)-(9) may be isolated by either crystallization or sublimation. All products are solids at ordinary temperatures. They can be readily characterized by ${ }^{1} \mathrm{H}$ NMR and IR spectroscopy (Table 3 ). The latter method not only gives some insight into the bonding of the CO groups [20,21], but can also be used to distinguish between cis and trans arrangements in compounds of the type $\mathrm{M}^{\prime}\left(\mathrm{O}^{\mathrm{t}} \mathrm{Bu}\right)_{3} \mathrm{In}-\mathrm{M}^{\mathrm{T}}(\mathrm{CO})_{4}-\mathrm{In}\left(\mathrm{O}^{1} \mathrm{Bu}\right)_{3} \mathrm{M}^{\prime}$, which have a local symmetry of either $C_{2 \mathrm{v}}$ or $D_{4 \mathrm{~h}}$ at the transition metal, $\mathrm{M}^{\mathrm{T}}[22,23]$. Table 3 lists only $A_{1}$ stretching frequencies, which are assigned to the carbonyl group situated trans to the $\mathbf{M}^{\prime}-\mathbf{M}^{\mathbf{T}}$ bond [24].

\subsection{Reactivity of $n \mathrm{~s}^{2}$-configurated main group elements towards transition metals}

Comparison of the reaction times and temperatures in Table 2 reveals distinct differences in the thermal reaction of metal carbonyls with metal alkoxides. If the $n \mathrm{~s}^{2}$ element is constant the reactivity trend $\mathrm{Ni}(\mathrm{CO})_{4}>\mathrm{Fe}_{2}(\mathrm{CO})_{9}>\mathrm{Mo}(\mathrm{CO})_{6}>$ $\mathrm{Cr}(\mathrm{CO})_{6}>\mathrm{W}(\mathrm{CO})_{6}$ can be established and is consistent with established results of other substitution reactions [20]. On the other hand, there is also a pronounced 
TABLE 2

Synthetic procedures for mixed main group metal alkoxide transition metal carbonyl complexes $(\mathrm{R}=$ tert-butyl $)$

\begin{tabular}{|c|c|}
\hline Compound and abbreviations & Conditions and yield ${ }^{a}$ \\
\hline $\mathrm{Tl}(\mathrm{OR})_{3} \mathrm{Sn}-\mathrm{Cr}(\mathrm{CO})_{5}(\mathrm{TSC})$ & $(\mathrm{OC})_{5} \mathrm{Cr} * \mathrm{THF} / \mathrm{THF} / \mathrm{RT} / 12 \mathrm{~h} / \mathrm{S} / 50 \%$ \\
\hline $\mathrm{Tl}(\mathrm{OR})_{3} \mathrm{Sn}-\mathrm{Mo}(\mathrm{CO})_{5}(\mathrm{TSM})$ & $\mathrm{Mo}(\mathrm{CO})_{6} / \mathrm{T} / 105^{\circ} \mathrm{C} / 5 \mathrm{~h} / \mathrm{S} / 70 \%$ \\
\hline $\mathrm{Tl}(\mathrm{OR})_{3} \mathrm{Sn}-\mathrm{W}(\mathrm{CO})_{5}(\mathrm{TSW})$ & $(\mathrm{OC})_{5} \mathrm{~W}^{*} \mathrm{THF} / \mathrm{THF} / \mathrm{RT} / 12 \mathrm{~h} / \mathrm{S} / 47 \%$ \\
\hline $\mathrm{Sn}(\mathrm{OR})_{3} \mathrm{In}-\mathrm{Cr}(\mathrm{CO})_{5}(\mathrm{SIC})$ & $\mathrm{Cr}(\mathrm{CO})_{6} / \mathrm{T} / 110^{\circ} \mathrm{C} / 40 \mathrm{~h} / \mathrm{S} / 35 \%$ \\
\hline $\mathrm{Sn}(\mathrm{OR})_{3} \mathrm{In}-\mathrm{Mo}(\mathrm{CO})_{3}(\mathrm{SIM})$ & $\mathrm{Mo}(\mathrm{CO})_{6} / \mathrm{T} / 80^{\circ} \mathrm{C} / 5 \mathrm{~h} / \mathrm{S} / 39 \%$ \\
\hline $\mathrm{Sn}(\mathrm{OR})_{3} \mathrm{In}-\mathrm{W}(\mathrm{CO})_{5}(\mathrm{SIW})$ & $\mathrm{W}(\mathrm{CO})_{6} / \mathrm{T} / 95^{\circ} \mathrm{C} / 2 \mathrm{~d} / \mathrm{S} / 17 \%$ \\
\hline $\mathrm{Sn}(\mathrm{OR})_{3} \mathrm{In}-\mathrm{Fe}(\mathrm{CO})_{4}(\mathrm{SIF})$ & $\mathrm{Fe}_{2}(\mathrm{CO})_{6} / \mathrm{T} / \mathrm{RT} / 3 \mathrm{~h} /-/$ quant \\
\hline $\mathrm{Ge}(\mathrm{OR})_{3} \mathrm{In}-\mathrm{Cr}(\mathrm{CO})_{5}(\mathrm{GIC})$ & $\mathrm{Cr}(\mathrm{CO})_{6} / \mathrm{T} / 85^{\circ} \mathrm{C} / 13 \mathrm{~h} / \mathrm{S} / 41 \%$ \\
\hline $\mathrm{Ge}(\mathrm{OR})_{3} \mathrm{In}-\mathrm{Mo}(\mathrm{CO})_{5}(\mathrm{GIM})$ & $\mathrm{Mo}(\mathrm{CO})_{6} / \mathrm{T} / 80^{\circ} \mathrm{C} / 3 \mathrm{~h} / \mathrm{S} / 45^{\circ} \%$ \\
\hline $\mathrm{Ge}(\mathrm{OR})_{3} \operatorname{In}-\mathrm{W}(\mathrm{CO})_{5}(\mathbf{G I W})$ & $\mathrm{W}(\mathrm{CO})_{6} / \mathrm{T} / 90^{\circ} \mathrm{C} / 1 \mathrm{~d} /-/ \mathrm{n} . \mathrm{d}$ \\
\hline$(\mathrm{OC})_{5} \mathrm{Cr}-\mathrm{In}(\mathrm{OR})_{3} \mathrm{Sn}-\mathrm{Cr}(\mathrm{CO})_{5}(\mathrm{CISC})$ & $(\mathbf{S I C})+(\mathrm{OC})_{5} \mathrm{Cr} * \mathrm{THF} / \mathrm{THF} / \mathrm{RT} / 24 \mathrm{~h} / \mathrm{C} / 80 \%$ \\
\hline$(\mathrm{OC})_{5} \mathrm{Mo}-\mathrm{In}(\mathrm{OR})_{3} \mathrm{Sn}-\mathrm{Mo}(\mathrm{CO})_{5}(\mathrm{MISM})$ & $2 \mathrm{Mo}(\mathrm{CO})_{6} / \mathrm{T} / 80^{\circ} \mathrm{C} / 11 \mathrm{~h} / \mathrm{C} / 83 \%$ \\
\hline$(\mathrm{OC})_{5} \mathrm{Mo}-\mathrm{In}(\mathrm{OR})_{3} \mathrm{Sn}-\mathrm{Cr}(\mathrm{CO})_{5}(\mathrm{MISC})$ & $(\mathrm{SIM})+(\mathrm{OC})_{5} \mathrm{Cr} * \mathrm{THF} / \mathrm{THF} / \mathrm{RT} / 24 \mathrm{~h} / \mathrm{C} / 75 \%$ \\
\hline$(\mathrm{OC})_{5} \mathrm{Cr}-\mathrm{In}(\mathrm{OR})_{3} \mathrm{Sn}-\mathrm{Mo}(\mathrm{CO})_{5}(\mathrm{CISM})$ & $(\mathrm{SIC})+(\mathrm{OC})_{5} \mathrm{Mo}^{*} \mathrm{THF} / \mathrm{THF} / \mathrm{RT} / 24 \mathrm{~h} / \mathrm{C} / 58 \%$ \\
\hline$(\mathrm{OC})_{5} \mathrm{Fe}-\mathrm{In}(\mathrm{OR})_{2} \mathrm{Sn}-\mathrm{Cr}(\mathrm{CO})_{5}($ FISC $)$ & $(\mathrm{SIF})+(\mathrm{OC})_{5} \mathrm{Cr} * \mathrm{THF} / \mathrm{THF} / \mathrm{RT} / 24 \mathrm{~h} / \mathrm{C} / 45 \%$ \\
\hline$(\mathrm{OC})_{3} \mathrm{Ni}-\mathrm{In}(\mathrm{OR})_{3} \mathrm{Sn}-\mathrm{Ni}(\mathrm{CO})_{3}(\mathrm{NISN})$ & $2 \mathrm{Ni}(\mathrm{CO})_{4} / \mathrm{T} / \mathrm{RT} / 1 \mathrm{~h} / \mathrm{C} / \mathrm{n} . \mathrm{d}$ \\
\hline$(\mathrm{OC})_{5} \mathrm{Cr}-\operatorname{In}(\mathrm{OR})_{3} \mathrm{Ge}-\mathrm{Cr}(\mathrm{CO})_{5}(\mathrm{CIGC})$ & $2 \mathrm{Cr}(\mathrm{CO})_{6} / \mathrm{T} / 90^{\circ} \mathrm{C} / 56 \mathrm{~h} / \mathrm{C} / \mathrm{n} . \mathrm{d}$ \\
\hline$(\mathrm{OC})_{5} \mathrm{Mo}-\mathrm{In}(\mathrm{OR})_{3} \mathrm{Ge}-\mathrm{Mo}(\mathrm{CO})_{5}(\mathrm{MIGM})$ & $2 \mathrm{Cr}(\mathrm{CO})_{6} / \mathrm{T} / 80^{\circ} \mathrm{C} / 8 \mathrm{~h} / \mathrm{C} / 75 \%$ \\
\hline$(\mathrm{OC})_{5} \mathrm{Mo}-\mathrm{In}(\mathrm{OR})_{3} \mathrm{Ge}-\mathrm{Cr}(\mathrm{CO})_{5}(\mathrm{MIGC})$ & $(\mathbf{G I M})+(\mathrm{OC})_{5} \mathrm{Cr} * \mathrm{THF} / \mathrm{THF} / \mathrm{RT} / 24 \mathrm{~h} / \mathrm{C} / 70 \%$ \\
\hline$(\mathrm{OC})_{5} \mathrm{~W}-\mathrm{In}(\mathrm{OR})_{3} \mathrm{Ge}-\mathrm{Cr}(\mathrm{CO})_{5}(\mathrm{WIGC})$ & $(\mathbf{G I W})+\mathrm{Cr}(\mathrm{OC})_{6} / \mathrm{T} / 90^{\circ} \mathrm{C} / 60 \mathrm{~h} / \mathrm{C} / 62 \%$ \\
\hline$(\mathrm{OC})_{4} \mathrm{Fe}-\mathrm{In}(\mathrm{OR})_{3} \mathrm{Ge}-\mathrm{Fe}(\mathrm{CO})_{4}$ (FIGF) & $2 \mathrm{Fe}_{2}(\mathrm{CO})_{9} / \mathrm{T} / \mathrm{RT} / 11 \mathrm{~h} / \mathrm{C} / 48 \%$ \\
\hline$(\mathrm{OC})_{5} \mathrm{Mo}-\mathrm{In}(\mathrm{OR})_{3} \mathrm{Ge}-\mathrm{Fe}(\mathrm{CO})_{4}(\mathrm{MIGF})$ & $(\mathbf{G I M})+\mathrm{Fe}_{2}(\mathrm{CO})_{9} / \mathrm{T} / \mathrm{RT} / 10 \mathrm{~h} / \mathrm{C} / 65 \%$ \\
\hline$(\mathrm{OC})_{3} \mathrm{Ni}-\ln (\mathrm{OR})_{3} \mathrm{Ge}-\mathrm{Ni}(\mathrm{CO})_{3}(\mathrm{NIGN})$ & $2 \mathrm{Ni}(\mathrm{CO})_{4} / \mathrm{T} /-20^{\circ} \mathrm{C} / 1 \mathrm{~h} / \mathrm{C} / 70 \%$ \\
\hline trans- $\left[\mathrm{Tl}(\mathrm{OR})_{3} \mathrm{Sn}\right]_{2} \mathrm{Cr}(\mathrm{CO})_{4}(\mathrm{TSCST})$ & $\mathrm{nbd} \mathrm{Cr}(\mathrm{CO})_{4} / \mathrm{BF} / 80^{\circ} \mathrm{C} / 2 \mathrm{~d} / \mathrm{C} / 40 \%$ \\
\hline trans $-\left[\mathrm{Tl}(\mathrm{OR})_{3} \mathrm{Sn}\right]_{2} \mathrm{Mo}(\mathrm{CO})_{4}(\mathrm{TSMST})$ & $\mathrm{nbd} \mathrm{Mo}(\mathrm{CO})_{4} / \mathrm{BF} / 70^{\circ} \mathrm{C} / 1 \mathrm{~d} / \mathrm{C} / 73 \%$ \\
\hline$c i s-\left[\mathrm{Sn}(\mathrm{OR})_{3} \mathrm{In}\right]_{2} \mathrm{Cr}(\mathrm{CO})_{4}(\mathbf{S I C I S})$ & $\mathrm{nbd} \mathrm{Cr}(\mathrm{CO})_{4} / \mathrm{BF} / 70^{\circ} \mathrm{C} / 1 \mathrm{~d} / \mathrm{C} / 72 \%$ \\
\hline$\left[\mathrm{Sn}(\mathrm{OR})_{3} \mathrm{In}\right]_{2} \mathrm{Mo}(\mathrm{CO})_{4}(\mathrm{SIMIS})$ & $\mathrm{nbd} \mathrm{Mo}(\mathrm{CO})_{4} / \mathrm{BF} / 65^{\circ} \mathrm{C} / 1 \mathrm{~d} / \mathrm{C} / 84 \%$ \\
\hline$c i s-\left[\mathrm{Sn}(\mathrm{OR})_{3} \mathrm{In}\right]_{2} \mathrm{~W}(\mathrm{CO})_{4}(\mathrm{SIWIS})$ & $\mathrm{nbd} \mathrm{W}(\mathrm{CO})_{4} / \mathrm{BF} / 80^{\circ} \mathrm{C} / 2 \mathrm{~d} / \mathrm{C} / 75 \%$ \\
\hline$c i s-\left[\mathrm{Ge}(\mathrm{OR})_{3} \mathrm{In}\right]_{2} \mathrm{Cr}(\mathrm{CO})_{4}(\mathrm{GICIG})$ & nbd $\mathrm{Cr}(\mathrm{CO})_{4} / \mathrm{BF} / 70^{\circ} \mathrm{C} / 1 \mathrm{~d} / \mathrm{C} / 89 \%$ \\
\hline $\begin{array}{l}c i s-\left[(\mathrm{OC})_{5} \mathrm{Cr}-\mathrm{Sn}(\mathrm{OR})_{3} \mathrm{In}\right]_{2} \mathrm{Cr}(\mathrm{CO})_{4} \\
(\mathrm{CSICISC})\end{array}$ & $(\mathrm{SICIS})+2(\mathrm{OC})_{5} \mathrm{Cr} * \mathrm{THF} / \mathrm{THF} / \mathrm{RT} / 24 \mathrm{~h} / \mathrm{C} / 75 \%$ \\
\hline $\begin{array}{l}{\left[(\mathrm{OC})_{5} \mathrm{Cr}-\mathrm{Sn}(\mathrm{OR})_{3} \mathrm{In}\right]_{2} \mathrm{Mo}(\mathrm{CO})_{4}} \\
\text { (CSIMISC) }\end{array}$ & $(\mathrm{SIMIS})+2(\mathrm{OC})_{5} \mathrm{Cr} * \mathrm{THF} / \mathrm{THF} / \mathrm{RT} / 24 \mathrm{~h} / \mathrm{C} / 61 \%$ \\
\hline$(\mathrm{OC})_{4} \mathrm{Fe}-\mathrm{Sn}(\mathrm{OR})_{3} \mathrm{Sr}(\mathrm{OR})_{3} \mathrm{Sn}-\mathrm{Fe}(\mathrm{CO})_{4}$ & $2 \mathrm{Fe}_{2}(\mathrm{CO})_{9} / \mathrm{T} / \mathrm{RT} / 20 \mathrm{~h} / \mathrm{C} / 36 \%$ \\
\hline & \\
\hline $\begin{array}{l}(\mathrm{OC})_{5} \mathrm{Cr}-\mathrm{Sn}(\mathrm{OR})_{3} \mathrm{Eu}(\mathrm{OR})_{3} \mathrm{Sn}-\mathrm{Cr}(\mathrm{CO})_{5} \\
(\mathrm{CSESC})\end{array}$ & $2 \mathrm{Cr}(\mathrm{CO})_{6} / \mathrm{B} / 80^{\circ} \mathrm{C} / 5 \mathrm{~h} / \mathrm{C} / 48 \%$ \\
\hline $\begin{array}{l}(\mathrm{OC})_{5} \mathrm{Cr}-\mathrm{Sn}(\mathrm{OR})_{3} \mathrm{Ba}(\mathrm{OR})_{3} \mathrm{Sn}-\mathrm{Cr}(\mathrm{CO})_{5} \\
(\mathrm{CSBSC})\end{array}$ & $2 \mathrm{Cr}(\mathrm{CO})_{6} / \mathrm{B} / 80^{\circ} \mathrm{C} / 60 \mathrm{~h} / \mathrm{C} / 41 \%$ \\
\hline $\begin{array}{l}(\mathrm{OC})_{5} \mathrm{Cr}-\mathrm{Sn}(\mathrm{OR})_{3} \mathrm{Sr}(\mathrm{OR})_{3} \mathrm{Sn}-\mathrm{Cr}(\mathrm{CO})_{5} \\
\text { (CSSSC) }\end{array}$ & $2 \mathrm{Cr}(\mathrm{CO})_{6} / \mathrm{T} / 100^{\circ} \mathrm{C} / 17 \mathrm{~h} / \mathrm{D} / 88 \%$ \\
\hline
\end{tabular}


TABLE 2 (continued)

$\begin{array}{ll}{\left[\mathrm{Co}(\mathrm{OR})_{4} \mathrm{Sn}-\mathrm{Fe}(\mathrm{CO})_{4}\right]_{2}(\mathrm{FSCCSF})} & 2 \mathrm{Fe}_{2}(\mathrm{CO})_{9} / \mathrm{T} / \mathrm{RT} / 18 \mathrm{~h} / \mathrm{C} / 44 \% \\ (\mathrm{OC})_{4} \mathrm{Fe}-\mathrm{Sn}(\mathrm{OR})_{3} \mathrm{~Pb}(\mathrm{OR})_{3} \mathrm{Sn}-\mathrm{Fe}(\mathrm{CO})_{4} & 2 \mathrm{Fe}_{2}(\mathrm{CO})_{9} / \mathrm{T} / \mathrm{RT} / 12 \mathrm{~h} / \mathrm{C} / 84 \% \\ (\mathrm{FSPSF}) & \\ {\left[(\mathrm{RO})_{2} \mathrm{GeORFe}(\mathrm{CO})_{4}\right]_{2} \mathrm{~Pb}(\mathrm{GFPFG})} & 2 \mathrm{Fe}_{2}(\mathrm{CO})_{9} / \mathrm{T} / \mathrm{RT} / 2 \mathrm{~h} / \mathrm{C} / 95 \% \\ & \\ & 2 \mathrm{Ni}(\mathrm{CO})_{4} / \mathrm{T} / 5^{\circ} \mathrm{C} / 30 \mathrm{~min} / \mathrm{D} / 97 \% \\ {\left[\mathrm{Na}\left(\mathrm{O}^{\mathrm{t} B u}\right)_{3} \mathrm{Ge}-\mathrm{Ni}(\mathrm{CO})_{3}\right]_{2}(\mathrm{NGN})^{\mathrm{b}}} & 2 \mathrm{Ni}(\mathrm{CO})_{4} / \mathrm{T} / 12^{\circ} \mathrm{C} / 30 \mathrm{~min} / \mathrm{D} / 94 \% \\ {\left[\mathrm{Na}\left(\mathrm{O}^{\mathrm{t} B u}\right)_{3} \mathrm{Sn}-\mathrm{Ni}(\mathrm{CO})_{3}\right]_{2}(\mathrm{NSN})^{\mathrm{b}}} & 2 \mathrm{Mo}(\mathrm{CO})_{6} / \mathrm{T} / 80^{\circ} \mathrm{C} / 3 \mathrm{~h} / \mathrm{D} / 95 \% \\ {\left[\mathrm{Na}\left(\mathrm{O}^{\mathrm{t} B u}\right)_{3} \mathrm{Ge}-\mathrm{Mo}(\mathrm{CO})_{5}\right]_{2}(\mathrm{NGM})^{\mathrm{b}}} & 2 \mathrm{Mo}(\mathrm{CO})_{6} / \mathrm{T} / 85^{\circ} \mathrm{C} / 3 \mathrm{~h} / \mathrm{D} / 93 \% \\ {\left[\mathrm{Na}\left(\mathrm{O}^{\mathrm{t} B u}\right)_{3} \mathrm{Sn}-\mathrm{Mo}(\mathrm{CO})_{5}\right]_{2}(\mathrm{NSM})^{\mathrm{b}}} & \end{array}$

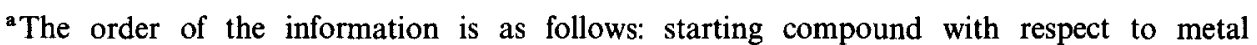
carbonyl/solvent $\left(\mathrm{B}=\right.$ benzene, $\mathrm{T}=$ toluene, $\mathrm{BF}=$ benzine-fraction (b.p. $\left.100-140^{\circ} \mathrm{C}\right), \mathrm{THF}=$ tetrahydrofuran)/reaction temperature $(\mathrm{RT}=$ room temperature $) /$ reaction time/method of separation $(\mathrm{C}=$ crystallization, $\mathrm{S}=$ sublimation, $\mathrm{D}=$ deposition $) / \mathrm{yield}$ in $\%$ (n.d. = not determined). ${ }^{b}$ Data to be published elsewhere.

difference in reactivity at the $n s^{2}$ element and the following series has been assessed: In(I) $\gg \mathrm{Ge}(\mathrm{II})>\mathrm{Sn}(\mathrm{II})>\mathrm{Tl}(\mathrm{I}), \mathrm{Pb}(\mathrm{II})$.

We have been unsuccessful in preparing $\mathrm{Tl}(\mathrm{I})$ or $\mathrm{Pb}$ (II) complexes of transition metals, although some complexes have been obtained with different ligands at these metal atoms $[25,26]$. For example, $\mathrm{Tl}\left(\mathrm{O}^{t} \mathrm{Bu}\right)_{3} \mathrm{Sn}$ will exclusively react with $\mathrm{Mo}(\mathrm{CO})_{6}$ to give $\mathrm{Tl}\left(\mathrm{O}^{t} \mathrm{Bu}\right)_{3} \mathrm{Sn}-\mathrm{Mo}(\mathrm{CO})_{5}$. No evidence of the substitution product formed by complexation at the thallium atom is obtained from the IR and NMR spectra of the reaction mixture. The $\mathrm{Sn}-\mathrm{Mo}$ bond can be unambiguously established by an X-ray structure determination [11].

The indium(I) centre in the "Janus-Head" molecules of the type $\operatorname{In}\left(\mathrm{Ot}^{t} \mathrm{Bu}\right)_{3} \mathrm{M}^{\prime}$ $\left(\mathrm{M}^{\prime}=\mathrm{Ge}, \mathrm{Sn}\right)$ is much more reactive than the germanium or tin centres. For example, the two isomers $(\mathrm{CO})_{5} \mathrm{Cr}-\mathrm{In}\left(\mathrm{O}^{\prime} \mathrm{Bu}\right)_{3} \mathrm{Sn}-\mathrm{Mo}(\mathrm{CO})_{5} \quad(\mathrm{CISM})$ and $(\mathrm{CO})_{5} \mathrm{Mo}-$ $\mathrm{In}\left(\mathrm{O}^{\mathrm{t}} \mathrm{Bu}\right)_{3} \mathrm{Sn}-\mathrm{Cr}(\mathrm{CO})_{5}$ (MISC) can be synthesized separately as displayed in eqns. (10) and (11) and are found to have distinctly different physical properties and different IR data (see Table 3) [11].

$$
\begin{aligned}
& \operatorname{In}\left(\mathrm{O}^{t} \mathrm{Bu}\right)_{3} \mathrm{Sn}+\mathrm{Cr}(\mathrm{CO})_{6} \rightarrow(\mathrm{CO})_{5} \mathrm{Cr}-\operatorname{In}\left(\mathrm{O}^{\mathrm{t}} \mathrm{Bu}\right)_{3} \mathrm{Sn}+\mathrm{CO} \\
& (\mathrm{CO})_{5} \mathrm{Cr}-\operatorname{In}\left(\mathrm{O}^{\mathrm{t} B u}\right)_{3} \mathrm{Sn}+\mathrm{Mo}(\mathrm{CO})_{6} \rightarrow(\mathrm{CO})_{5} \mathrm{Cr}-\mathrm{In}\left(\mathrm{O}^{\mathrm{t} B u}\right)_{3} \mathrm{Sn}-\mathrm{Mo}(\mathrm{CO})_{5}+\mathrm{CO}
\end{aligned}
$$

(CISM)

$$
\begin{aligned}
& \operatorname{In}\left(\mathrm{O}^{t} \mathrm{Bu}\right)_{3} \mathrm{Sn}+\mathrm{Mo}(\mathrm{CO})_{6} \rightarrow(\mathrm{CO})_{5} \mathrm{Mo}-\mathrm{In}\left(\mathrm{O}^{\mathrm{t} B u}\right)_{3} \mathrm{Sn}+\mathrm{CO} \\
& (\mathrm{CO})_{5} \mathrm{Mo}-\mathrm{In}\left(\mathrm{O}^{\mathrm{t}} \mathrm{Bu}\right)_{3} \mathrm{Sn}+\mathrm{Cr}(\mathrm{CO})_{6} \rightarrow(\mathrm{CO})_{5} \mathrm{Mo}-\mathrm{In}\left(\mathrm{O}^{\mathrm{t}} \mathrm{Bu}\right)_{3} \mathrm{Sn}-\mathrm{Cr}(\mathrm{CO})_{5}+\mathrm{CO}
\end{aligned}
$$

\section{(MISC)}

The structures of MISC and CISM have been confirmed by X-ray crystallography, revealing structural differences. CISM crystallizes in the orthorhombic space group 
TABLE 3

${ }^{1} \mathrm{H}$ NMR $(\delta, \mathrm{ppm})$ and IR [CO stretch trans to $\mathrm{M}^{\mathrm{T}}-\mathrm{M}$ bond or $\mathrm{M}(\mathrm{CO})_{n}$ group in $\mathrm{cm}^{-1}$ ]

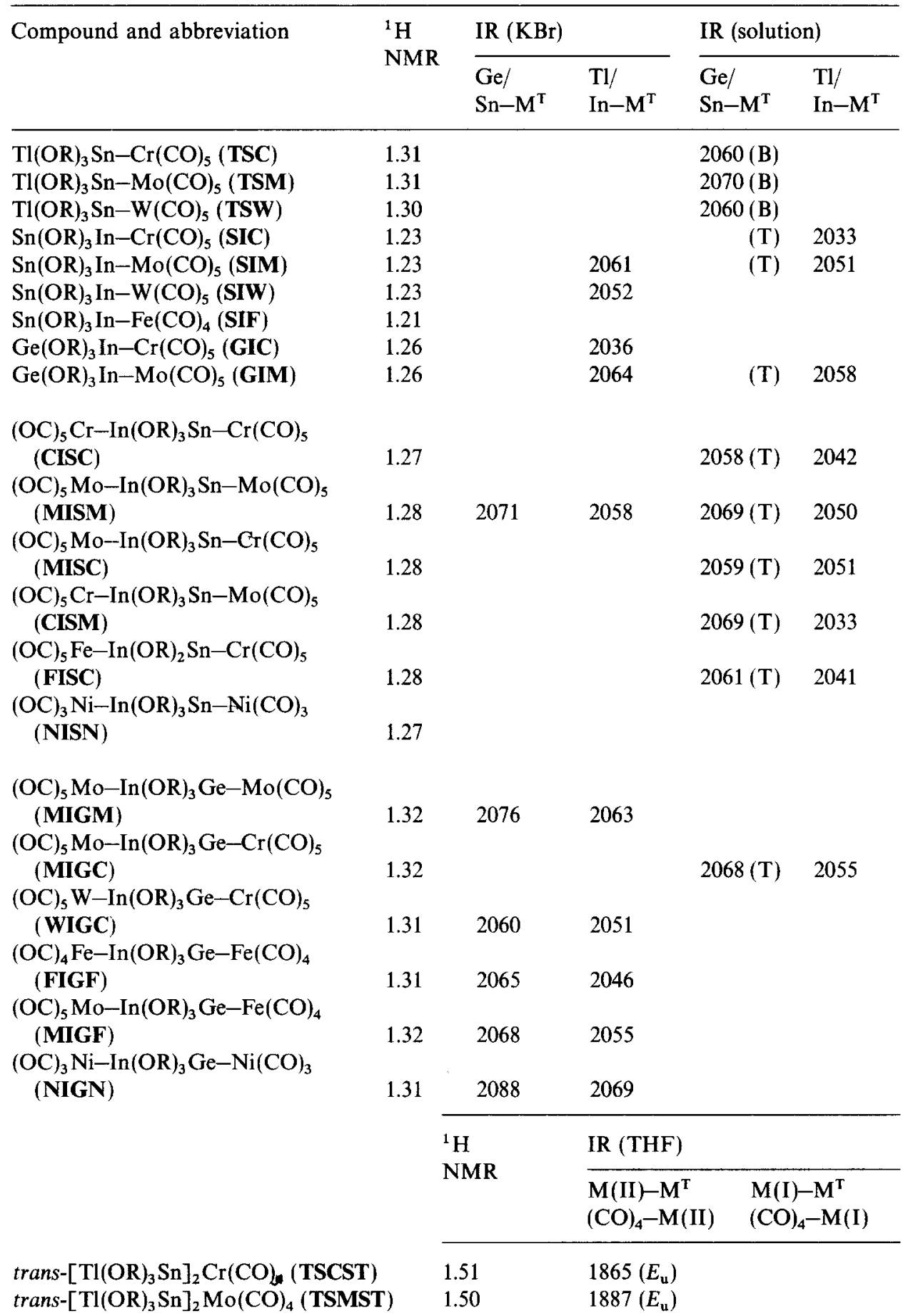


TABLE 3 (continued)

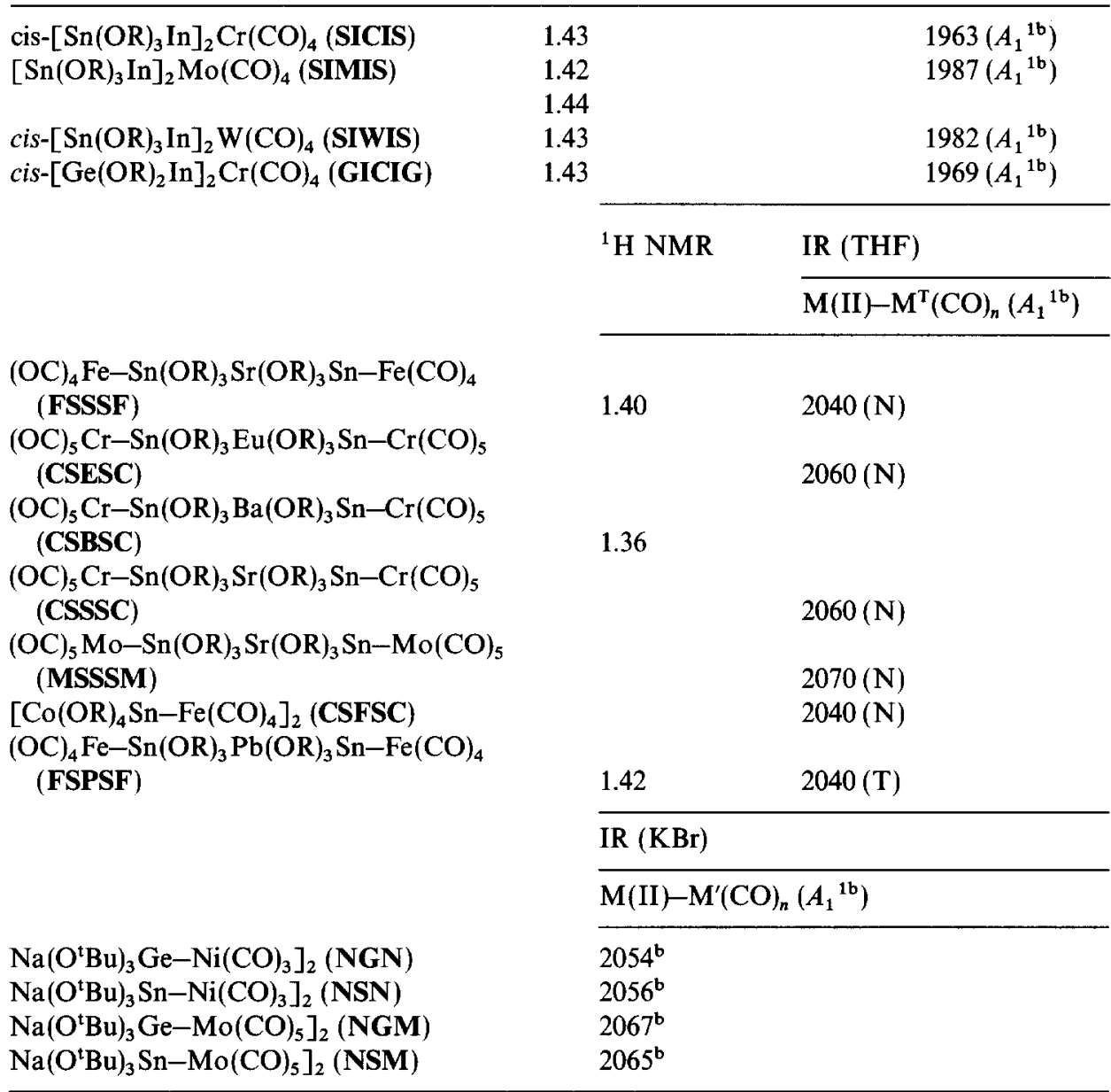

${ }^{\mathrm{a}} \mathrm{B}=$ benzene; $\mathrm{N}=$ nujol; $\mathrm{T}=$ toluene; $\mathrm{THF}=$ tetrahydrofuran; $\mathrm{R}=$ tert -butyl.

${ }^{\mathrm{b}}$ Data to be published elsewhere.

Pnma $[a=14.93(2), b=14.33(2), c=15.46(2), Z=4]$ and MISC in the monoclinic space group $P 2_{1} / n\left[a=10.51(1), b=30.30(2), c=10.748(7), \beta=107.36(7)^{\circ}, Z=\right.$ 4] [11].

The indium atom is more reactive towards metal carbonyls than germanium or tin in $\operatorname{In}\left(\mathrm{O}^{t} \mathrm{Bu}\right)_{3} \mathrm{M}^{\prime}$. This may be explained by the fact that the ${ }^{\mathrm{t}} \mathrm{BuO}$ group has a formal charge of -1 , and the two metals have charges $\mathrm{In}+1$ and $\mathrm{Ge} / \mathrm{Sn}+2$. The indium atom should therefore be more basic than germanium or tin. Conversely, elements within the Periodic Table tend to become less basic with increasing atomic mass, which is often explained by a more diffuse character of the orbitals of heavy main group elements [27]. Hence In(I) should be less basic than Ge(II). From our 
experimental results, it seems that the charge effect is more important than the size effect.

3. STRUCTURES, SPECTROSCOPY AND BONDING

3.1. Structural characterization of $n s^{2}$-configurated main group metal alkoxide transition metal carbonyl complexes

A number of metal carbonyl complexes of mixed metal alkoxides have been characterized by X-ray crystallography. Table 4 lists crystal data not available elsewhere [28]. Table 5 lists selected molecular distances [28] together with those of the non-complexed alkoxides $\operatorname{In}\left(\mathrm{O}^{t} \mathrm{Bu}\right)_{3} \mathrm{Sn}$ (IS), $\mathrm{Tl}\left(\mathrm{O}^{\mathrm{t}} \mathrm{Bu}\right)_{3} \mathrm{Sn}$ (TS), $\mathrm{Sn}\left(\mathrm{O}^{t} \mathrm{Bu}\right)_{3}$ $\mathrm{Pb}\left(\mathrm{O}^{t} \mathrm{Bu}\right)_{3} \mathrm{Sn}$ (SPS) and $\mathrm{Ge}\left(\mathrm{O}^{t} \mathrm{Bu}\right)_{3} \mathrm{Ca}\left(\mathrm{O}^{\mathrm{t}} \mathrm{Bu}\right)_{3} \mathrm{Ge}$ (GCG). In Fig. 2 the data for the complexes have been arranged in a diagram with increasing $\mathbf{M}^{\prime} \cdots M$ distance. Drawings of the molecular structures are displayed in Figs. 3-9. The labelling of carbon atoms and carbonyl oxygen atoms has been omitted for clarity.

As can be seen from the figures, all metallic elements in the molecules are arranged in one-dimensional chains, with some of the metal atoms displaying direct metal-metal bonding. Apart from the direct metal-metal bonds, some of the metals are held close together by three oxygen bridges which are disposed outside the metal chain and which originate from the coordination of the alkoxide groups. Whereas in most of the compounds the one-dimensional array of metal atoms is linear, some compounds show a bend angle of $96-99^{\circ}$ at the central transition metal [GICIG, 95.8 $(1)^{\circ}$; CSICISC, $98.6(3)^{\circ}$, CSIMISC, 97.9 $\left.(3)^{\circ}\right]$. This is due to the fact that bissubstitution at the central pseudo-octahedral transition metal is in cis positions. As we have noted before [29], the distortion from linearity in FSPSF (in a simplified model) is due to the central metallic element $\mathrm{Pb}$, which has a $6 \mathrm{~s}^{2}$ configuration (stereochemical activity of the lone pair).

The reason for the difference in structure between the bent cis-compound GICIG and the linear trans-compound TSMST is unclear. As the trigonal pyramidally coordinated $n \mathrm{~s}^{2}$ elements are poorer $\pi$-acceptors than carbon monoxide, the substitution of a second ligand should occur in a cis position [30-33]. On the other hand, steric requirements also play an important part in ligand distributions around a metallic element and, owing to the bulkiness of the "Janus-Head" molecules, a trans arrangement should be favoured. Examination of trans-TSMST and cis-GICIG, -CSICISC and -CSIMISC reveals that the cone angle $\mathrm{O}-\mathrm{M}^{\prime}-\mathrm{O}$ at the main group element bound to the transition metal and the $\mathbf{M}^{\prime}-\mathrm{O}$ distance are different. For TSMST the mean $\mathrm{O}-\mathrm{Sn}-\mathrm{O}$ angle $=84.3^{\circ}[\mathrm{Sn}-\mathrm{O}=2.04(3) \AA]$ whereas for GICIG $\mathrm{O}-\mathrm{In}-\mathrm{O}=66.3^{\circ} \quad[\mathrm{In}-\mathrm{O}=2 \cdot 24(1) \AA]$, for CSICISC $\mathrm{O}-\mathrm{In}-\mathrm{O}=68.5^{\circ} \quad[\mathrm{In}-\mathrm{O}=$ $2.26(2) \AA]$ and for CSIMISC O-In-O $=67.7^{\circ}[\mathrm{In}-\mathrm{O}=2.27(2) \AA]$. Therefore, the indium atom can be characterized as much softer and more flexibile for geometrical constraints compared with tin, a classification we have already found by comparing 
TABLE 4

Crystal data for some new metal carbonyl complexes of mixed metal alkoxides [28]

\begin{tabular}{|c|c|c|c|c|c|c|}
\hline Compound & $\begin{array}{l}\text { Crystal } \\
\text { system }\end{array}$ & $\begin{array}{l}\text { Space } \\
\text { group }\end{array}$ & $\begin{array}{l}\text { Lattice } \\
\text { constants } \\
\AA{ }^{\circ}\end{array}$ & $\begin{array}{l}\text { Molecules } \\
\text { per unit } \\
(Z)\end{array}$ & $R\left(R_{\mathrm{w}}\right)^{\mathrm{b}}$ & $\begin{array}{l}\text { Sym- } \\
\text { metry }\end{array}$ \\
\hline $\begin{array}{l}\mathrm{C}_{21} \mathrm{H}_{27} \mathrm{CrFeInO}_{12} \mathrm{Sn} \\
\text { (FISC) }\end{array}$ & Monoclinic & $P 2_{1} / n$ & $\begin{array}{ll}a & 12.122(9) \\
b & 18.646(19) \\
c & 13.666(9) \\
\beta & 92.07(8)\end{array}$ & 4 & $\begin{array}{l}0.0776 \\
(0.0678)\end{array}$ & $C_{1}$ \\
\hline $\begin{array}{l}\mathrm{C}_{22} \mathrm{H}_{27} \mathrm{GeInMo}_{2} \mathrm{O}_{13} \\
\text { (MIGM) }\end{array}$ & Monoclinic & $P 2_{1} / n$ & $\begin{array}{ll}a & 10.470(9) \\
b & 30.40(2) \\
c & 10.720(9) \\
\beta & 107.36(8)\end{array}$ & 4 & $\begin{array}{l}0.0707 \\
(0.0704)\end{array}$ & $C_{1}$ \\
\hline $\begin{array}{l}\mathrm{C}_{28} \mathrm{H}_{54} \mathrm{CrGe}_{2} \mathrm{In}_{2} \mathrm{O}_{10} \\
\text { (GICIG) }\end{array}$ & Monoclinic & $P 2_{1} / n$ & $\begin{array}{ll}a & 10.137(8) \\
b & 26.30(2) \\
c & 16.030(9) \\
\beta & 91.22(2)\end{array}$ & 4 & $\begin{array}{l}0.0637 \\
(0.0632)\end{array}$ & $C_{1}$ \\
\hline $\begin{array}{l}\mathrm{C}_{28} \mathrm{H}_{54} \mathrm{MoO}_{10} \mathrm{Sn}_{2} \mathrm{Tl}_{2} \\
\text { (TSMST) }^{\mathrm{T}}\end{array}$ & Tetragonal & $P 4_{2} / n$ & $\begin{array}{ll}a & 20.65(2) \\
c & 10.029(8)\end{array}$ & 4 & 0.1273 & $C_{\mathrm{i}}$ \\
\hline $\begin{array}{l}\mathrm{C}_{32} \mathrm{H}_{54} \mathrm{Fe}_{2} \mathrm{O}_{14} \mathrm{Sn}_{2} \mathrm{Sr} \\
\text { (FSSSF) }\end{array}$ & Monoclinic & $\mathrm{P} 2_{1} / n$ & $\begin{array}{ll}a & 10.598(5) \\
b & 13.681(8) \\
c & 15.982(8) \\
\beta & 97.06(4)\end{array}$ & 2 & $\begin{array}{l}0.0507 \\
(0.0476)\end{array}$ & $C_{\mathrm{i}}$ \\
\hline $\begin{array}{l}\mathrm{C}_{34} \mathrm{H}_{54} \mathrm{BaCr}_{2} \mathrm{O}_{16} \mathrm{Sn}_{2} \\
\text { (CSBSC) }\end{array}$ & Monoclinic & $P 2_{1} / n$ & $\begin{array}{ll}a & 15.408(9) \\
b & 10.014(8) \\
c & 15.799(9) \\
\beta & 98.39(5)\end{array}$ & 2 & $\begin{array}{l}0.0463 \\
(0.0460)\end{array}$ & $C_{\mathrm{i}}$ \\
\hline $\begin{array}{l}\mathrm{C}_{38} \mathrm{H}_{54} \mathrm{Cr}_{3} \mathrm{In}_{2} \mathrm{O}_{20} \mathrm{Sn}_{2} \\
\text { (CSICISC) }\end{array}$ & Triclinic & $P \overline{1}$ & $\begin{array}{ll}a & 13.714(9) \\
b & 16.102(13) \\
c & 16.244(9) \\
\alpha & 114.43(7) \\
\beta & 112.87(6) \\
\gamma & 97.12(7)\end{array}$ & 2 & $\begin{array}{l}0.0796 \\
(0.0763)\end{array}$ & $C_{1}$ \\
\hline $\begin{array}{l}\mathrm{C}_{38} \mathrm{H}_{54} \mathrm{Cr}_{2} \mathrm{In}_{2} \mathrm{MoO}_{20} \mathrm{Sn}_{2} \\
\text { (CSIMISC) }^{\mathrm{a}}\end{array}$ & Triclinic & $P \overline{1}$ & $\begin{array}{lr}a & 13.78(2) \\
b & 16.13(4) \\
c & 16.29(4) \\
\alpha & 114.0(1) \\
\beta & 112.6(1) \\
\gamma & 97.6(1)\end{array}$ & 2 & $\begin{array}{l}0.0973 \\
(0.0954)\end{array}$ & $C_{1}$ \\
\hline
\end{tabular}

${ }^{a}$ Bad crystalline material: crystals of TSMST might be twinned.

${ }^{b} R$ value $\left(R_{W}=\right.$ weighted $\left.R\right)$ of structure refinement. 
TABLE 5

The most pertinent molecular dimensions $(\AA)$ in some metal carbonyl complexes of mixed metal alkoxides [28]

\begin{tabular}{llllll}
\hline Compound & $\mathrm{M} \cdots \mathrm{M}$ & $\mathrm{In}-\mathrm{M}(\mathrm{T})$ & $\mathrm{Ge} / \mathrm{Sn}-\mathrm{M}(\mathrm{T})$ & $\mathrm{M}-\mathrm{O}$ & $\mathrm{Ge} / \mathrm{Sn}-\mathrm{O}$ \\
\hline MIGM & $2.954(2)$ & $2.702(4)$ & $2.567(4)$ & $2.244(9)$ & $1.842(9)$ \\
GICIG & $2.962(2)$ & $2.563(4)$ & - & $2.236(9)$ & $1.872(8)$ \\
FISC & $3.046(1)$ & $2.420(9)$ & $2.574(9)$ & $2.187(20)$ & $2.034(20)$ \\
MISM [11] & $3.078(1)$ & $2.742(1)$ & $2.761(1)$ & $2.141(6)$ & $2.122(9)$ \\
MISC [11] & $3.087(2)$ & $2.734(2)$ & $2.636(3)$ & $2.213(9)$ & $2.067(9)$ \\
CSICISC & $3.116(2)$ & $2.571(3)$ & $2.596(6)$ & $2.263(15)$ & $2.025(12)$ \\
CSIMISC & $3.123(3)$ & $2.710(5)$ & $2.592(6)$ & $2.271(17)$ & $2.003(13)$ \\
FSSSF & $3.292(1)$ & & $2.491(2)$ & $2.550(6)$ & $2.027(6)$ \\
TSM [11] & $3.298(1)$ & & $2.770(2)$ & $2.557(15)$ & $2.017(9)$ \\
TSMST & $3.320(3)$ & & $2.687(5)$ & $2.568(50)$ & $2.036(30)$ \\
FSPSF [29] & $3.327(1)$ & & $2.476(1)$ & $2.593(20)$ & $2.016(9)$ \\
CSBSC & $3.493(1)$ & & $2.649(3)$ & $2.705(6)$ & $2.034(5)$ \\
& & & & \\
IS [11] & $3.200(3)$ & & & $2.413(4)$ & $2.032(4)$ \\
TS [10] & $3.306(3)$ & & & $2.595(7)$ & $2.023(9)$ \\
SPS [9] & $3.361(1)$ & & & $2.56(2)$ & $2.074(8)$ \\
GCG [9] & $2.999(2)$ & & & $2.36(1)$ & $1.903(3)$
\end{tabular}

${ }^{a} \mathrm{Ge}\left(\mathrm{O}^{\mathrm{t}} \mathrm{Bu}\right)_{3} \mathrm{Ca}\left(\mathrm{O}^{\mathrm{t}} \mathrm{Bu}\right)_{3} \mathrm{Ge}$ as reference for a normal $\mathrm{Ge}-\mathrm{O}$ bond length.

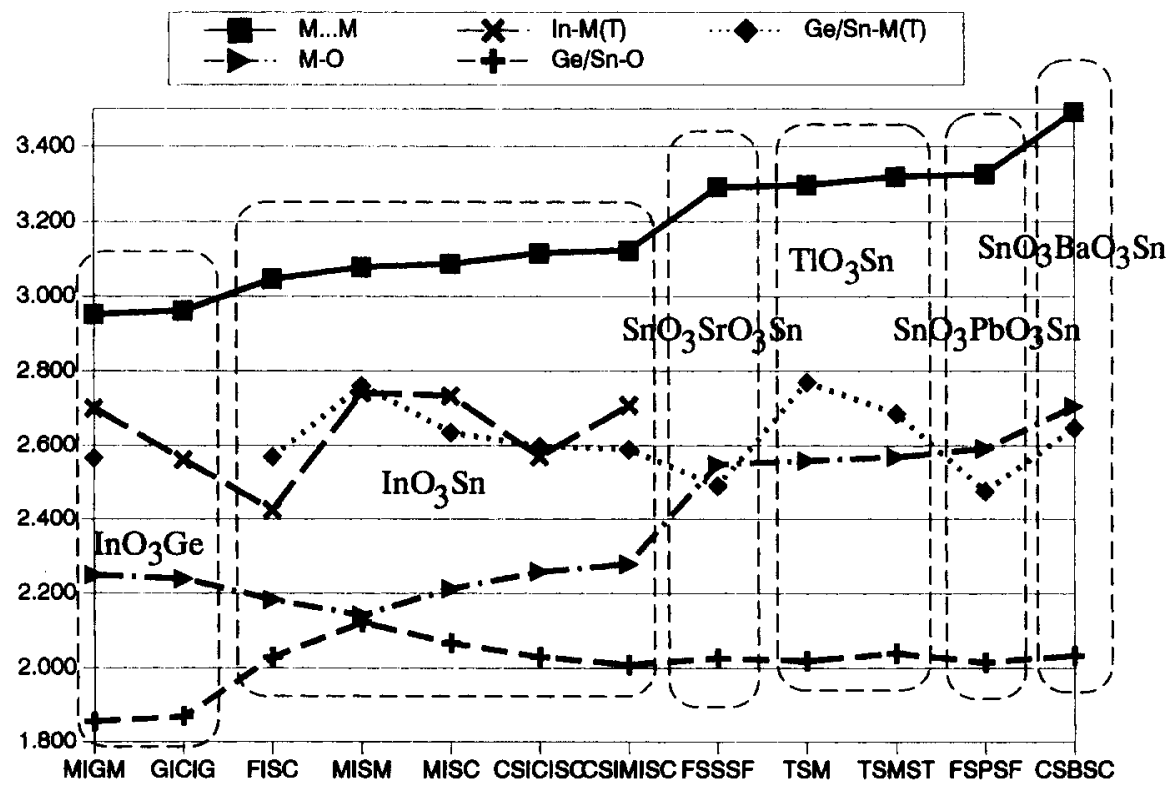

Fig. 2. Plot of different distances (in $\AA$ ) within metal alkoxide metal carbonyl complexes. The inner skeleton formulae of the alkoxides are used to separate different groups of compounds. For the designation of the compounds, see the text. 


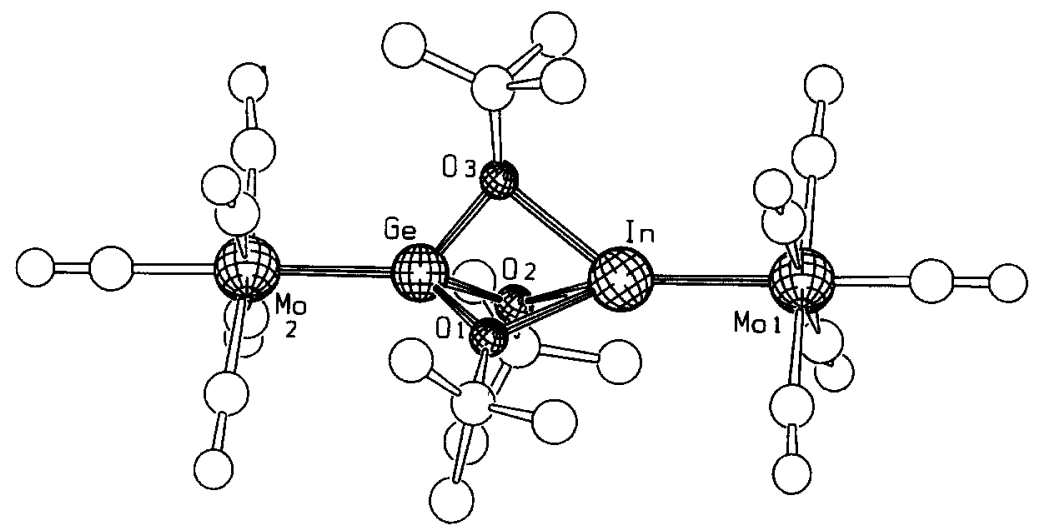

Fig. 3. Molecular structure of $(\mathrm{CO})_{5} \mathrm{Mo}-\mathrm{In}(\mathrm{OtBu})_{3} \mathrm{Ge}-\mathrm{Mo}(\mathrm{CO})_{5}(\mathrm{MIGM})$.

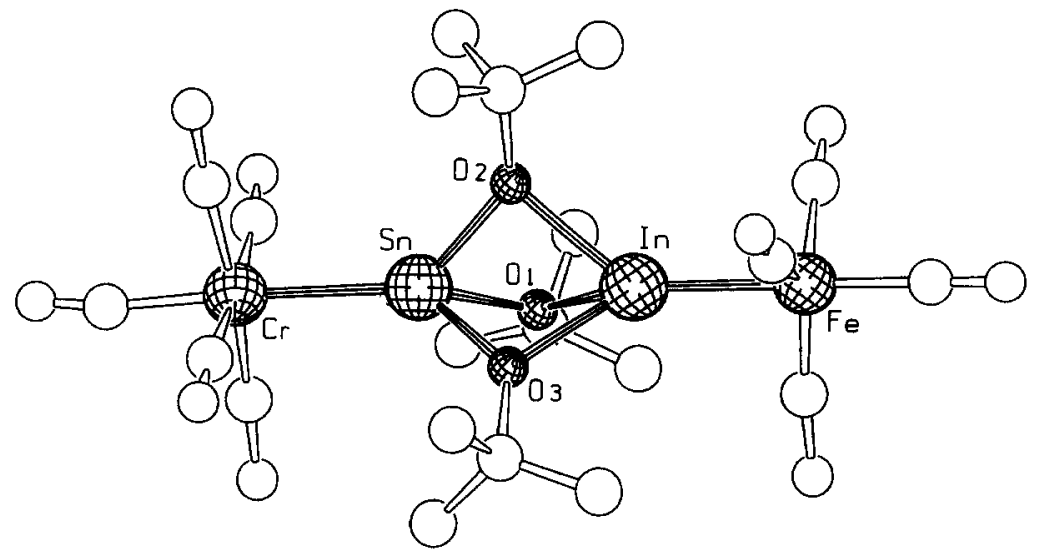

Fig. 4. Molecular structure of $(\mathrm{CO})_{4} \mathrm{Fe}-\mathrm{In}(\mathrm{OtBu})_{3} \mathrm{Sn}-\mathrm{Cr}(\mathrm{CO})_{5}($ FISC).

$\mathrm{Sn}(\mathrm{II})$ with $\mathrm{Ge}$ (II) or $\mathrm{Pb}$ (II) in mixed metal alkoxides [9]. The difference in structures between GICIG and TSMST may therefore be attributed to steric factors. The factors responsible for cis or trans arrangements in metal carbonyl complexes may be equilibrated in CSIMISC. In this case only, both cis and trans isomers can be detected by NMR in solution. This suggests that in some instances crystal packing or solubility may also play an important role.

Generally, the metal-metal distance in the trigonal bipyramidal cages $\mathrm{M}^{\prime}\left(\mathrm{O}^{t} \mathrm{Bu}\right)_{3} \mathrm{M}$ and $\mathrm{M}^{\prime}\left(\mathrm{O}^{t} \mathrm{Bu}\right)_{3} \mathrm{M}\left(\mathrm{O}^{\mathrm{t}} \mathrm{Bu}\right)_{3} \mathrm{M}^{\prime}$ along the threefold axis is decreased when transition metals coordinate to the $n \mathrm{~s}^{2}$ elements (Table 5). At the same time, the $\mathbf{M}^{\prime}-\mathrm{O}$ distances decrease. The drain of electron density from the main group metal to the transition metal is reflected by a shrinkage of the radius of the metallic atom. This generalization is valid for $\operatorname{In}(\mathrm{I})$ and $\mathrm{Ge}(\mathrm{II})$ in any of the compounds under discussion and also for $\mathrm{Sn}(\mathrm{II})$, with some restrictions, as can be seen by comparing 


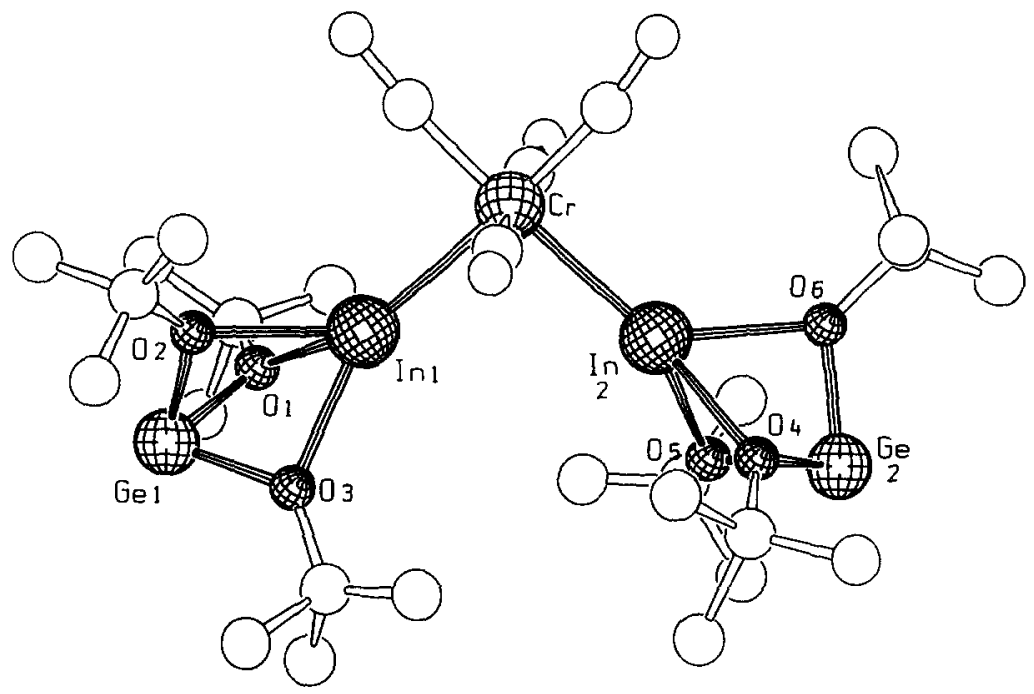

Fig. 5. Molecular structure of $\mathrm{Ge}(\mathrm{OtBu})_{3} \operatorname{In}-\mathrm{Cr}(\mathrm{CO})_{4}-\operatorname{In}(\mathrm{OtBu})_{3} \mathrm{Ge}$ (GICIG).

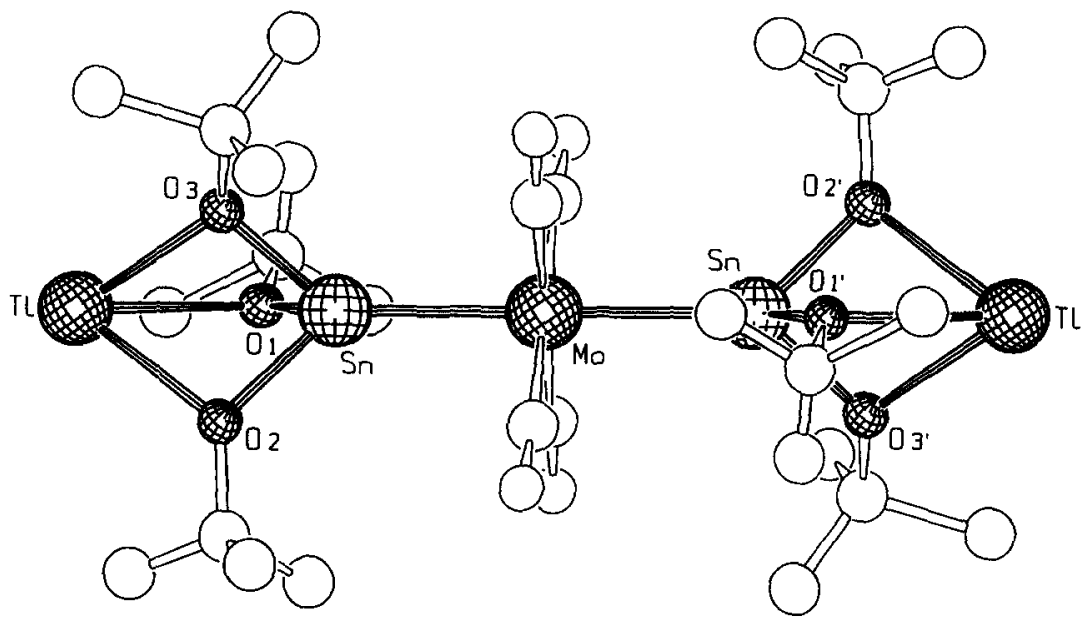

Fig. 6. Molecular structure of $\mathrm{Tl}(\mathrm{OtBu})_{3} \mathrm{Sn}-\mathrm{Mo}(\mathrm{CO})_{4}-\mathrm{Sn}(\mathrm{OtBu})_{3} \mathrm{Tl}$ (TSMST).

the distances in $\mathrm{Tl}\left(\mathrm{O}^{\mathrm{t}} \mathrm{Bu}\right)_{3} \mathrm{Sn}(\mathrm{TS})$ with $\mathrm{Tl}\left(\mathrm{O}^{\mathrm{t}} \mathrm{Bu}\right)_{3} \mathrm{Sn}-\mathrm{Mo}(\mathrm{CO})_{5}$ ( $\left.\mathrm{TSM}\right)$. However, there are also some exceptions. For example, the $\mathrm{Sn}-\mathrm{O}$ distances in MISC and MISM are longer than in the parent compound $\mathrm{Sn}\left(\mathrm{O}^{\mathrm{t}} \mathrm{Bu}\right)_{3} \mathrm{In}$ (see Table 5). To gain further understanding about this effect, we have selected some data from Table 5 and Fig. 2 and rearranged them in Fig. 10. All the compounds in Fig. 10 contain the bipyramid $\operatorname{In}\left(\mathrm{O}^{t} \mathrm{Bu}\right)_{3} \mathrm{Sn}$. The smallest distance between indium and tin is obtained in FISC and increases within the series MISM, MISC, CSICISC, CSIMISC. It can therefore be concluded that the coordination of iron and chromium at indium and 


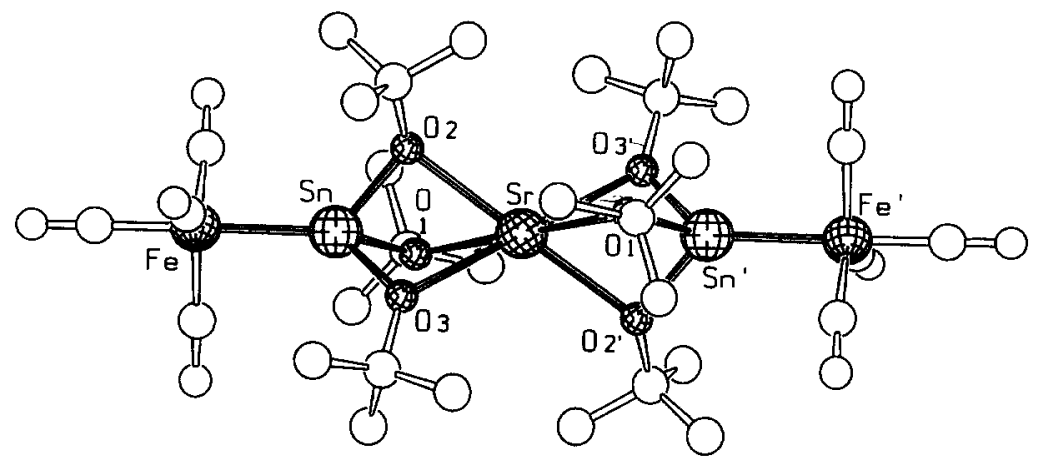

Fig. 7. Molecular structure of $(\mathrm{CO})_{4} \mathrm{Fe}-\mathrm{Sn}(\mathrm{OtBu})_{3} \mathrm{Sr}(\mathrm{OtBu})_{3} \mathrm{Sn}-\mathrm{Fe}(\mathrm{CO})_{4}(\mathrm{FSSSF})$.

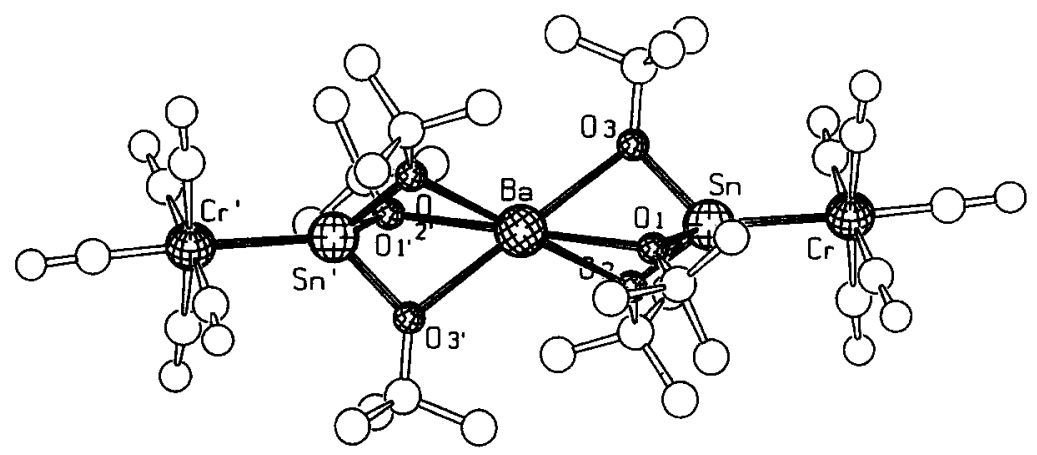

Fig. 8. Molecular structure of $(\mathrm{CO})_{5} \mathrm{Cr}-\mathrm{Sn}(\mathrm{OtBu})_{3} \mathrm{Ba}(\mathrm{OtBu})_{3} \mathrm{Sn}-\mathrm{Cr}(\mathrm{CO})_{5}$ (CSBSC).

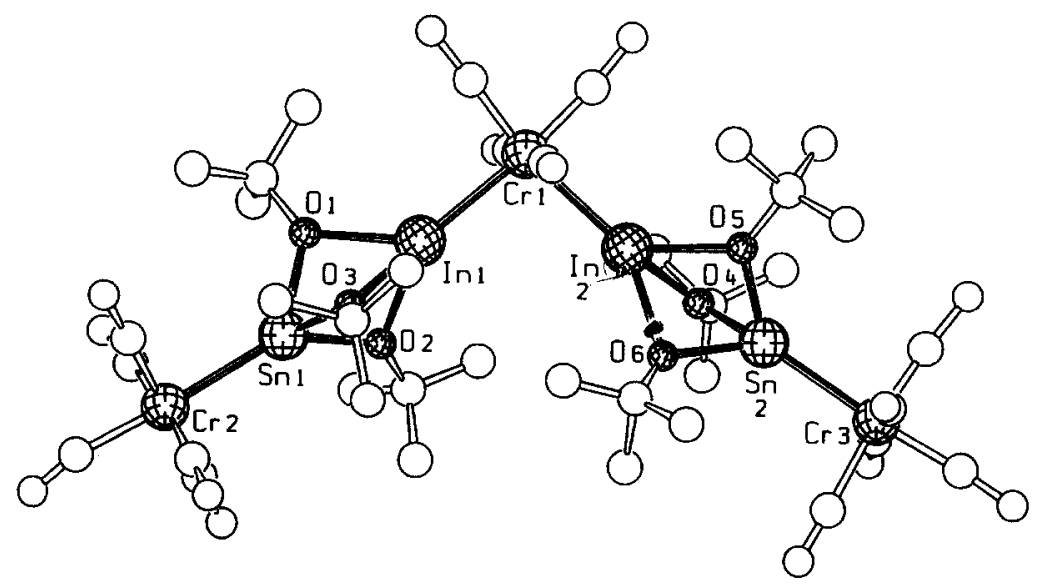

Fig. 9. Molecular structure of $(\mathrm{CO})_{5} \mathrm{Cr}-\mathrm{Sn}(\mathrm{OtBu})_{3} \mathrm{In}-\mathrm{Cr}(\mathrm{CO})_{4}-\mathrm{In}(\mathrm{OtBu})_{3} \mathrm{Sn}-\mathrm{Cr}(\mathrm{CO})_{5}(\mathrm{CSICISC})$. The compound CSIMISC is isotypic to CSICISC (see also Table 4). 


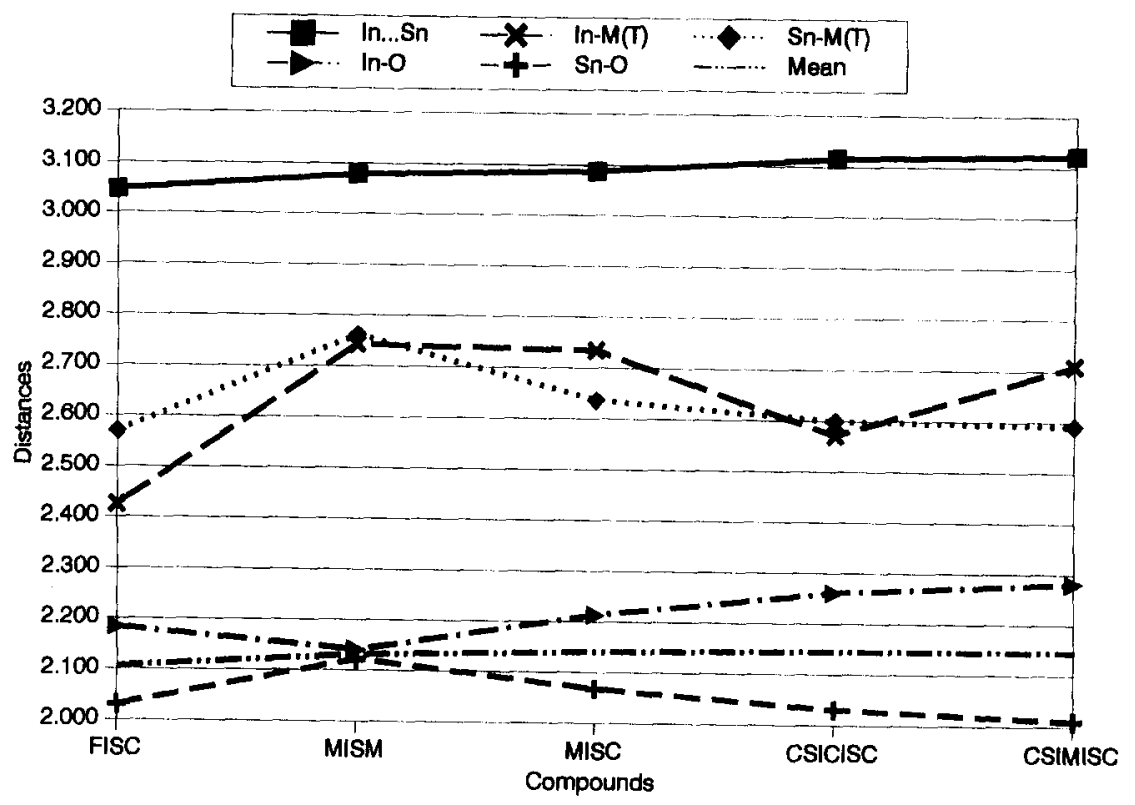

Fig. 10. Compounds containing $\operatorname{In}(\mathrm{OtBu})_{3} \mathrm{Sn}$ as metal alkoxide. The distances are given in $\AA$.

tin, respectively, is most effective with respect to the drain of electrons from the main group metals to the transition elements. The metal combinations $\mathrm{Mo}-\mathrm{Mo}$ or $\mathrm{Mo}-\mathrm{Cr}$ are less powerful than the combination $\mathrm{Fe}-\mathrm{Cr}$. In addition, the radii shrinkage is much more important at indium than germanium and tin, as can be concluded from the In-O distances in the metal carbonyl complexes compared with the free molecule $\operatorname{In}\left(\mathrm{O}^{t} \mathrm{Bu}\right)_{3} \mathrm{Sn}$. The mean shrinkage of the In-O distances is about $8 \%$ and that of the $\mathrm{Ge}-\mathrm{O}$ distances is $2.5 \%$. In complexes which contain only tin as the $n \mathrm{~s}^{2}$ element (FSSSF, FSPSF, CSBSC) the shrinkage is even less $(0.6 \%)$. This parallels our findings with respect to reactivity (see above), i.e. $\mathrm{In}$ (I) $\gg \mathrm{Ge}$ (II) $>\mathrm{Sn}$ (II).

Inspection of Table 5 and Fig. 10 shows that the two $n s^{2}$ elements $\operatorname{In}(\mathrm{I})$ and $\mathrm{Sn}$ (II) are not isolated from one another. The average of the In-O and $\mathrm{Sn}-\mathrm{O}$ distances for each compound parallels the In $\cdots$ Sn distance (Fig. 10). On the other hand, the $\mathrm{Sn}-\mathrm{O}$ distance may even increase with respect to $\operatorname{In}\left(\mathrm{O}^{t} \mathrm{Bu}\right)_{3}{ }^{\prime} \mathrm{Sn}$ (in MISM and MISC), but this increase is compensated for by an even higher shrinkage at the In site. These observations clearly show that the $\operatorname{In}\left(\mathrm{O}^{t} \mathrm{Bu}\right)_{3} \mathrm{Sn}$ part of the molecule is highly flexible and cage expansions on one side are followed by contractions on the other side and vice versa. Partial charge transfer within the cage may be conducted via the trigonal planar oxygen atoms or direct metal $\cdots$ metal interactions.

In Fig. 11 only those complexes with chromium bonded to tin are included. The $\mathrm{Cr}-\mathrm{Sn}$ distance within this series is constant, and the plot indicates that the most important contribution to the $\mathrm{Sn} \cdots \mathrm{M}$ distances comes from the metal atom (In, Ba) that is incorporated in the alkoxide cage. Thus the $\mathrm{M}-\mathrm{O}$ and $\mathrm{Sn} \cdots \mathrm{M}$ 


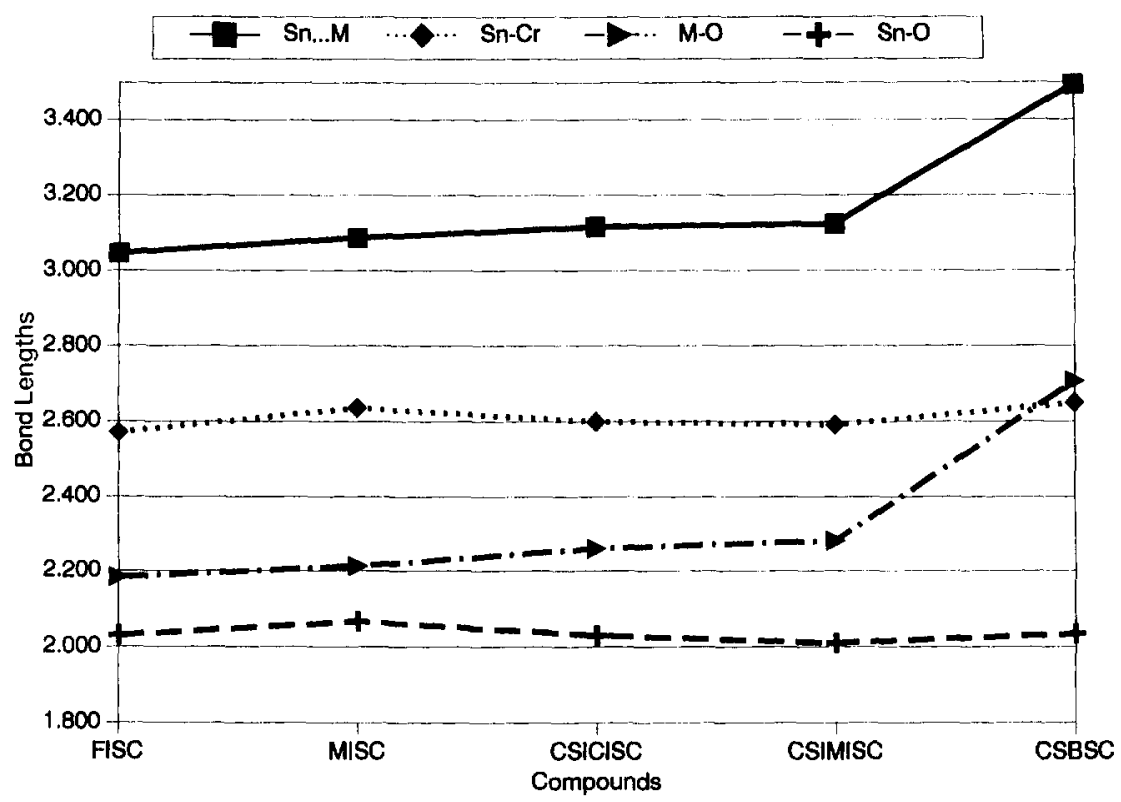

Fig. 11. Compounds with $\mathrm{Sn}-\mathrm{Cr}$ bonds. The bond lengths are given in $\AA$. The $\mathrm{Sn} \cdots \mathrm{M}$ curve and the $\mathrm{M} \cdots \mathrm{O}$ curve are almost parallel to one another.

curves are almost parallel to each other. As expected (Fig. 11), a transition metal atom coordinated to one $\operatorname{In}\left(\mathrm{O}^{t} \mathrm{Bu}\right)_{3} \mathrm{Sn}$ unit has a more important electron-attracting effect than the same transition metal with two $\mathrm{In}\left(\mathrm{O}^{\mathrm{t}} \mathrm{Bu}\right)_{3} \mathrm{Sn}$ cages attached (MISC versus CSIMISC).

Compounds of the spirocyclic system $\left({ }^{\mathrm{t}} \mathrm{BuO}\right) \mathrm{M}^{\prime}\left(\mathrm{O}^{\mathrm{t}} \mathrm{Bu}\right)_{2} \mathrm{M}\left(\mathrm{O}^{\mathrm{t}} \mathrm{Bu}\right)_{2}-$ $\mathrm{M}\left(\mathrm{O}^{\mathrm{t}} \mathrm{Bu}\right)_{2} \mathrm{M}^{\prime}\left(\mathrm{O}^{t} \mathrm{Bu}\right)$ can also be used as precursors for metal carbonyl complexes [13]. The iron carbonyl complex $(\mathrm{CO})_{4} \mathrm{Fe}-\mathrm{Sn}\left[\mathrm{O}^{t} \mathrm{Bu}\right]\left(\mathrm{O}^{t} \mathrm{Bu}\right)_{2} \mathrm{Co}\left(\mathrm{O}^{t} \mathrm{Bu}\right)_{2} \mathrm{Co}\left(\mathrm{O}^{t} \mathrm{Bu}\right)_{2^{-}}$ $\left[\mathrm{O}^{t} \mathrm{Bu}\right] \mathrm{Sn}-\mathrm{Fe}(\mathrm{CO})_{4}$ has been characterized by $\mathrm{X}$-ray crystallography. As in the compounds discussed above, the coordination of the iron carbonyl to the tin atom results in a decrease in the $\mathrm{Sn}-\mathrm{O}$ bond length and the $\mathrm{Sn} \cdots$ Co distance. Altogether six metallic elements are combined in the molecule in a one-dimensional fashion with an Fe-Sn $\cdots$ Co angle of $140.1^{\circ}$ [13].

\subsection{IR frequencies of $\mathrm{CO}$ in metal carbonyl complexes of mixed metal alkoxides}

The $A_{1}$ resonance in the IR spectra of substituted metal carbonyls of the type $\mathrm{M}\left(\mathrm{O}^{\mathrm{t}} \mathrm{Bu}\right)_{3} \mathrm{M}^{\prime}-\mathrm{M}^{\mathrm{T}}(\mathrm{CO})_{n}, \mathrm{M}^{\mathrm{T}}(\mathrm{CO})_{n}-\mathrm{M}\left(\mathrm{O}^{\mathrm{t}} \mathrm{Bu}\right)_{3} \mathrm{M}^{\prime}-\mathrm{M}^{\mathrm{T}}(\mathrm{CO})_{n}, \quad \mathrm{M}\left(\mathrm{O}^{\mathrm{t} B u}\right)_{3} \mathrm{M}^{\prime}-$ $\mathrm{M}^{\mathrm{T}}(\mathrm{CO})_{n} \mathrm{M}^{\prime}\left(\mathrm{O}^{\mathrm{t}} \mathrm{Bu}\right)_{3} \mathrm{M}$ or $(\mathrm{CO})_{n} \mathrm{M}^{\mathrm{T}}-\mathrm{M}^{\prime}\left(\mathrm{O}^{t} \mathrm{Bu}\right)_{3} \mathrm{M}\left(\mathrm{O}^{\prime} \mathrm{Bu}\right)_{3} \mathrm{M}^{\prime}-\mathrm{M}^{\mathrm{T}}(\mathrm{CO})_{n}$ may be used as a probe to distinguish between different substitution patterns in the "Janus-Head" molecules. Figure 12 shows that there are distinct regions in which the $v \mathrm{CO}$ stretch appears for $\mathrm{CO}$ trans to the $\mathrm{M}^{\prime}-\mathrm{M}^{\mathrm{T}}$ bond. As we have noted before, the $\mathrm{C}=\mathrm{O}$ stretch 


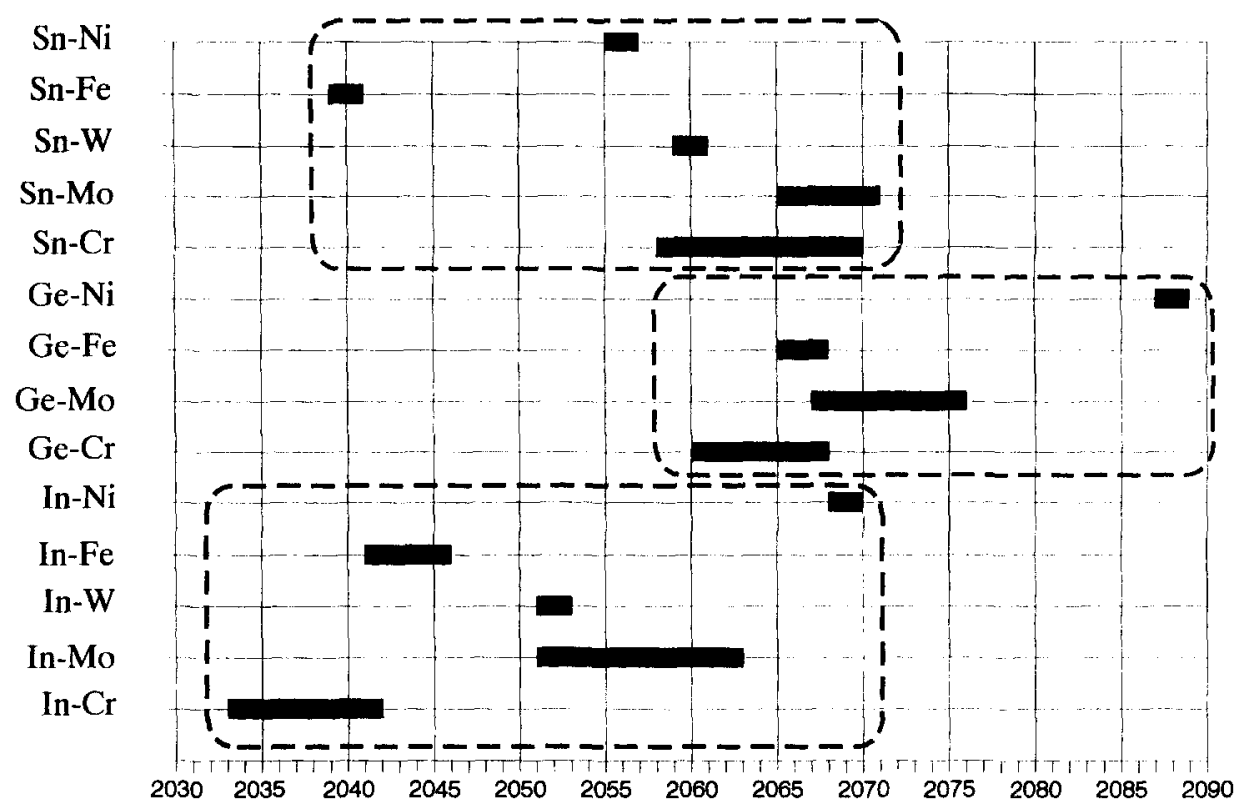

Fig. 12. CO absorption regions (wavenumbers, $\mathrm{cm}^{-1}$ ) of carbonyl groups trans to the metal-metal bonds indicated in the graph.

may be used as a probe of the extent of $\pi$-bonding to the metal carbonyl complex of mixed metal alkoxides. We would expect a shift to higher frequencies compared with standard THF complexes [23] for $\mathrm{CO}$ groups trans to the $\mathrm{M}^{\prime}-\mathrm{M}^{\mathrm{T}}$-bond if there is an important $M^{\prime} \leftarrow M^{\mathrm{T}}$ back-bonding via $\pi$-orbitals. From a consideration of the trans-carbonyl stretching frequencies in Table 3 it is clear that back-bonding from $\mathrm{M}^{\mathrm{T}}$ to $\mathrm{M}^{\prime}$ is present. All complexes with $n \mathrm{~s}^{2}$-metal donors seem to be reasonably strong $\sigma$-donors and weak to moderate $\pi$-acceptors. This can be concluded from the fact that alkylphosphine-substituted metal carbonyls have comparable wavenumbers $[34,35]$. Nevertheless, there are some interesting cases to be discussed in more detail. In Fig. 13 several compounds with similar substitution patterns have been assembled. Comparing the data for SIC and SIM, it is clear that an In-Mo bond should have more $\pi$-interaction than an $\mathrm{In}-\mathrm{Cr}$ bond as the wavenumber of the latter $A_{1} \mathrm{CO}$ vibration is $28 \mathrm{~cm}^{-1}$ less [neglecting the different experimental conditions (see Table 3)]. This effect is again observed (of the same magnitude) if GIC is compared with GIM. We therefore conclude that an In-Mo interaction is stronger than an In- $\mathrm{Cr}$ interaction. This interpretation finds its equivalent in molecular dimensions (see Fig. 2), in that all bonds adjacent to indium are much more effected by Mo coordination than by $\mathrm{Cr}$ coordination.

When the bidentate "Janus-Head" molecules MISC, MISM, MIGC and MIGM are compared with the monodentate species SIC, SIM, GIC and GIM, the resonance peaks of the $\mathrm{CO}$ vibrations trans to the $\mathrm{In}-\mathrm{Mo}$ bond are shifted to lower 


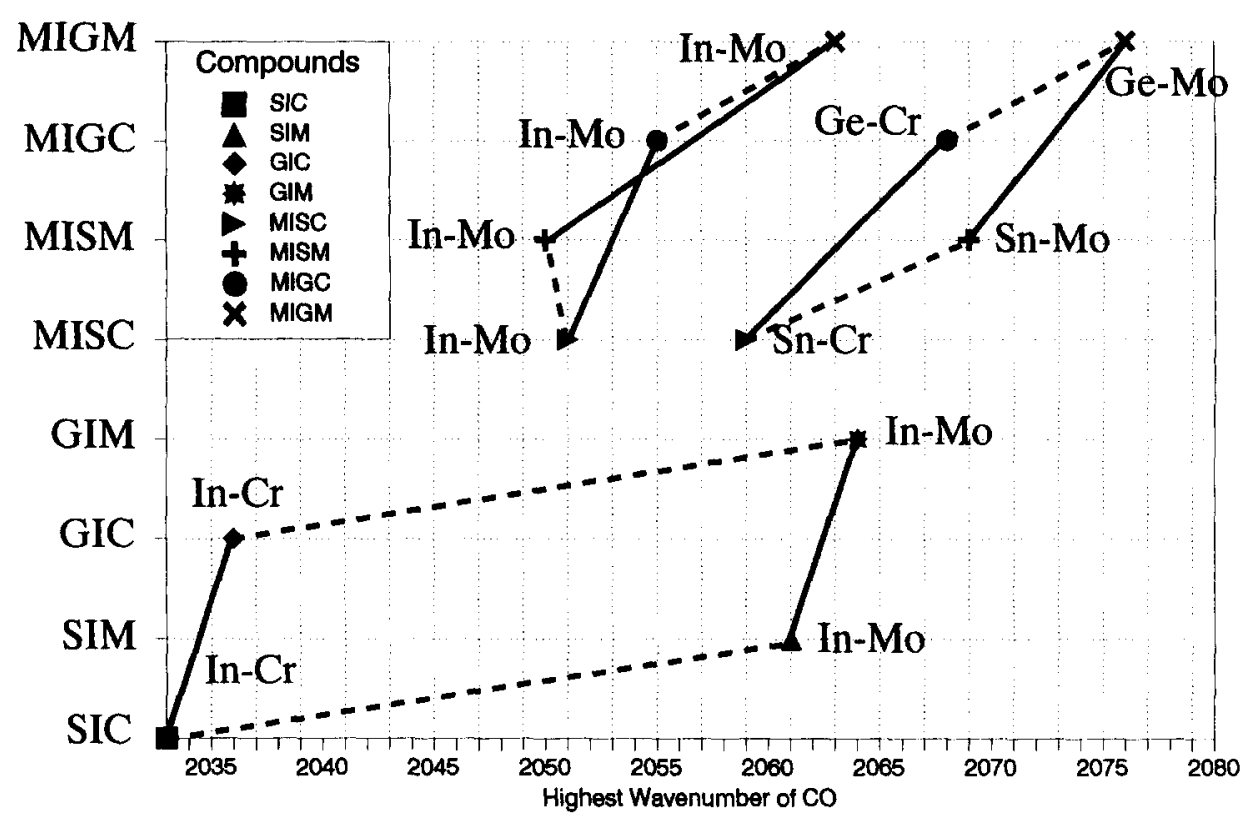

Fig. 13. Stretching frequencies (wavenumbers, $\mathrm{cm}^{-1}$ ) of the $\mathrm{CO}$ absorption trans to the indicated metalmetal bonds in compounds of the families $\mathrm{Sn}\left(\mathrm{O}^{\mathrm{t}} \mathrm{Bu}\right)_{3} \mathrm{In}-\mathrm{M}^{\mathrm{T}}(\mathrm{CO})_{5} \quad\left(\mathrm{M}^{\mathrm{T}}=\mathrm{Cr}, \quad \mathrm{Mo}\right)$ and $(\mathrm{CO})_{5} \mathrm{Mo}-\mathrm{In}\left(\mathrm{O}^{\mathrm{t}} \mathrm{Bu}\right)_{3} \mathrm{Sn}-\mathrm{M}^{\mathrm{T}}(\mathrm{CO})_{5}\left(\mathrm{M}^{\mathrm{T}}=\mathrm{Cr}, \mathrm{Mo}\right)$.

wavenumber (with exception of MIGM). This means that the second transition metal ligand entering the molecule at the Ge or Sn end has an impact also on the In-Mo bond. Again this is in accordance with our observation in the last section that bond lengths are affected by a second transition metal coordination at parts of the molecule which are not directly linked to the coordination centre. For the germanium or tin end of the molecules MISC, MISM, MIGC and MIGM, the CO groups at molybdenum again show higher wavenumbers than the $\mathrm{CO}$ groups attached to chromium, but the difference is much less than in the indium case. Also, the wavenumbers for $\mathrm{CO}$ trans to $\mathrm{Sn}-\mathrm{Cr}$ or $\mathrm{Sn}-\mathrm{Mo}$ are smaller than those which are trans to $\mathrm{Ge}-\mathrm{Cr}$ and $\mathrm{Ge}-\mathrm{Mo}$, respectively, indicating a more important $\pi$-backbonding at germanium than tin. With respect to the transition metals we may note that molybdenum results in stronger bonds to indium than chromium. As can be seen from Fig. 14, there is a dependence between the shift of the $\operatorname{CO}\left(A_{1}\right)$ trans to the $\mathrm{Cr}-\mathrm{Sn}$ bond and the $\mathrm{Sn} \cdots$ In distance. Interestingly the highest $\mathrm{CO}$ stretch is found for the shortest $\mathrm{Sn}$... In distance. Again, this may be taken as a criterion for an electron transfer along the metal-metal axis (directly or indirectly through the oxygen bridges of the alcoholate ligand). No correlation can be found for a similar plot of the $\mathrm{Sn}-\mathrm{Cr}$ distance versus the $\mathrm{CO}$ stretching frequency.

A qualitative MO model may be used to explain the different mesomeric effects in the carbonyl IR absorption of mixed metal alkoxide metal carbonyl complexes. 


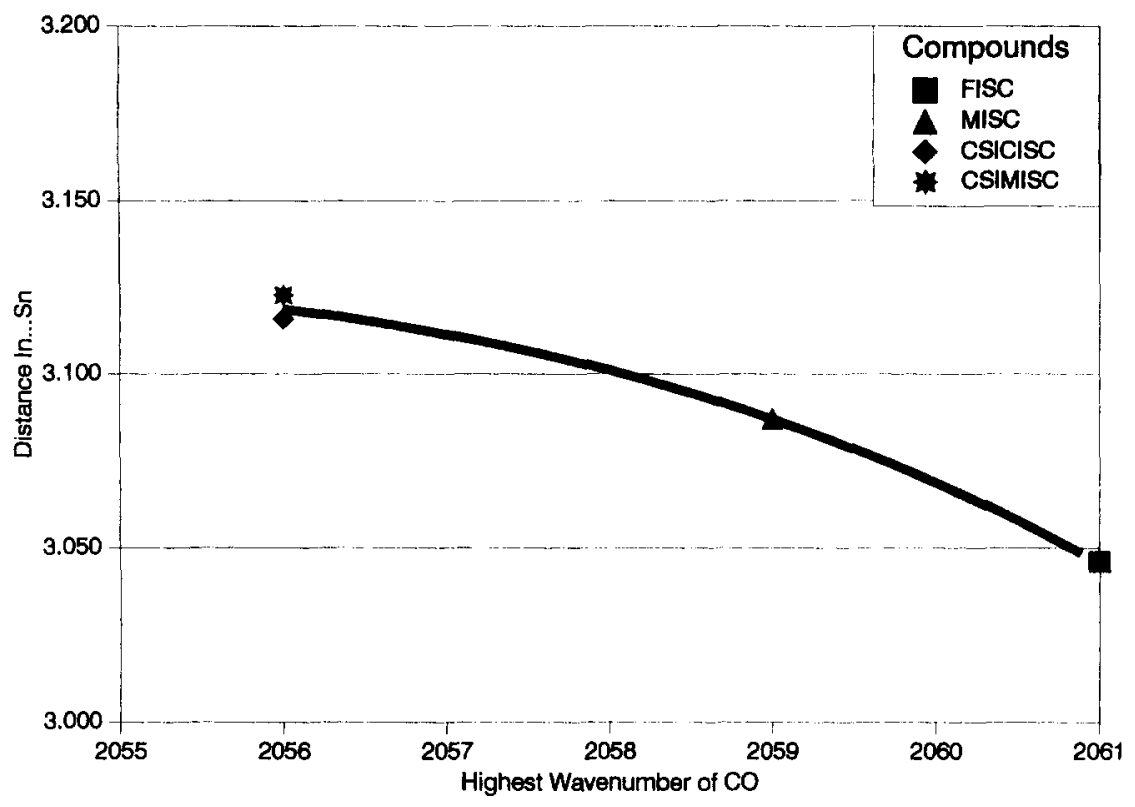

Fig. 14. Correlation of the $v(\mathrm{CO}) A_{1}$ band $\left(\mathrm{cm}^{-1}\right)$ with $\mathrm{In} \cdots$ Sn distance $(\AA)$ in selected compounds.

As the d-orbitals of the main group metals are high in energy compared with the d-orbitals of the transition metals, $\sigma^{*}$-orbitals of the mixed metal alkoxides must also be considered for back-bonding from the transition metal to the main group metal. The model would suggest an increase in length of the $\mathbf{M}^{\prime}-\mathrm{O}$ bonds when $\pi$ back-bonding is taking place through $\sigma^{*}$-orbitals, and no change in the $\mathrm{M}^{\prime}-\mathrm{O}$ distances when $\pi$-bonding is occurring through d-orbitals of the main group metals. As can be deduced from the experimental results, in many cases the inductive effects are dominant. In any case, Mo should be more appropriate than $\mathrm{Cr}$ for $\pi$-bonding as the energy levels of the $4 d$ orbitals match better the orbitals of $\operatorname{In}(\mathrm{I})$ and $\mathrm{Sn}$ (II), in agreement with the experiment (see above).

\subsection{Comments on a cluster concept}

So far we have considered the distance between metal atoms bridged by alkoxo groups as predominantly non-bonding. This fits in with the observation that all transition metal complexes of the $n \mathrm{~s}^{2}$-configurated metal alkoxides described above are colourless and may be classified as insulators. Nevertheless, a detailed look at the molecular dimensions (see above) reveals that the main group metals under consideration are very close to each other. Moreover, there are direct metal-metal bonds between the transition metals and the $n s^{2}$ elements. The one-dimensional metal chain therefore consists of di- or triatomic metal clusters separated by oxygen bridges. Within the metal chain the metallic elements are tightly linked or in loose 
contact. Restricted to the metal arrangement such systems have a "percolated" structure. There is a tiny step from these "percolated metal clusters" to conventional metal clusters, as exemplified by comparing the iron tetracarbonyl complexes of $\mathrm{Sn}(\mathrm{OtBu})_{3} \mathrm{~Pb}(\mathrm{OtBu})_{3} \mathrm{Sn}$ and $\mathrm{Ge}(\mathrm{OtBu})_{3} \mathrm{~Pb}(\mathrm{OtBu})_{3} \mathrm{Ge}$. These complexes are obtained when the lead stannate and germate are reacted with $\mathrm{Fe}_{2}(\mathrm{CO})_{9}$ [eqn.(12)] [29].

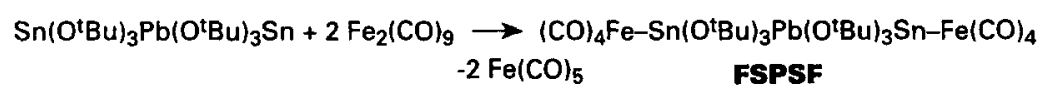

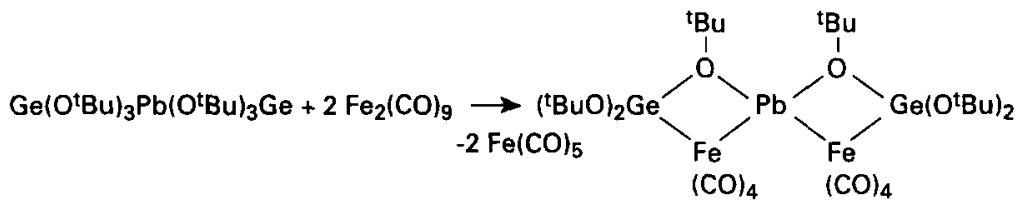

Whereas the ordinary compound FSPSF is formed in the case of the tin derivative, a molecule of similar constitution but of different structure is obtained for the germate. The core of the molecule consists of a cluster of five metal atoms linked by direct metal-metal bonds. A rearrangement of the ligands around the central lead atom is observed in the germanium compound with direct lead-iron bonding. The different structures of the germate and stannate complexes have been unambiguously characterized by X-ray crystallography [29]. Whereas FSPSF forms yellow crystals, its germanium analogue crystallizes in dark red needles. Of course, the metal alkoxide metal carbonyl complexes can also be used in thermal decomposition reactions as starting materials to classical cluster compounds. This is again exemplified by a reaction in which $\mathrm{Sn}(\mathrm{OtBu})_{3} \operatorname{InFe}(\mathrm{CO})_{4}(\mathrm{SIF})$ is thermally decomposed in a xylene solution [eqn. (13)] [36].

$$
\begin{aligned}
& 4 \mathrm{Sn}\left(\mathrm{O}^{t} \mathrm{Bu}\right)_{3} \mathrm{InFe}(\mathrm{CO})_{4} \rightarrow\left\{\left(\mathrm{In}_{2} \mathrm{O}\right)\left[\mathrm{Sn}\left(\mathrm{O}^{t} \mathrm{Bu}\right)_{2}\right]\left[\mathrm{Fe}(\mathrm{CO})_{4}\right]_{2}\right\}_{2}+\left[\mathrm{Sn}\left(\mathrm{O}^{t} \mathrm{Bu}\right)_{2}\right]_{2} \\
& +2^{\mathrm{t}} \mathrm{BuOH}+2\left(\mathrm{CH}_{3}\right)_{2} \mathrm{C}=\mathrm{CH}_{2}
\end{aligned}
$$

The high molecular mass product is deep yellow and has a central unit which is made up of an eight-membered $\mathrm{Fe}_{4} \mathrm{In}_{4}$ ring with direct $\mathrm{Fe}-\mathrm{In}$ bonds as in $\left[\left\{(\mathrm{CO})_{5} \mathrm{Mn}\right\} \operatorname{In}\left(\mu-\mathrm{Fe}(\mathrm{CO})_{4}\right)_{2} \operatorname{In}\left\{\mathrm{Mn}(\mathrm{CO})_{5}\right\}\right]$ [27]. The $\mathrm{Sn}\left(\mathrm{O}^{t} \mathrm{Bu}\right)_{2}$ units are coordinated to the system by oxygen metal bonds (X-ray structure).

\section{OLIGOMERS AND POLYMERS WITH ONE-DIMENSIONALLY ARRANGED METAL ATOMS}

We have shown in the preceding sections that transition metals may be used to link two metal alkoxides ("Janus-Head") by direct bonding and thus yield pentametallic (e.g. TSMST or GICIG) or heptametallic (e.g. CSICISC or CSIMISC) complexes. It is conceivable to use this sort of linkage in a repeated fashion in order to obtain higher molecular mass entities with one-dimensionally arranged metal 
atoms. Theoretically, there are several promising reaction pathways to obtain oligomers or polymers of the type $\left[\mathrm{M}^{\prime}\left(\mathrm{O}^{t} \mathrm{Bu}\right)_{3} \mathrm{M}-\mathrm{M}^{\mathrm{T}}(\mathrm{CO})_{m}\right]_{x}$, some of which are displayed in reactions (14)-(16).

$$
\begin{aligned}
& (\mathrm{CO})_{n} \mathrm{M}^{\mathrm{T}}-\mathrm{M}^{\prime}\left(\mathrm{O}^{\mathrm{t}} \mathrm{Bu}\right)_{3} \mathrm{M}-\mathrm{M}^{\mathrm{T}}(\mathrm{CO})_{n} \stackrel{\Delta}{\longrightarrow} \\
& \mathrm{M}(\mathrm{CO})_{n+1}+1 / x\left[-\mathrm{M}^{\prime}\left(\mathrm{O}^{\mathrm{t}} \mathrm{Bu}\right)_{3} \mathrm{M}-\mathrm{M}^{\mathrm{T}}(\mathrm{CO})_{n-1}-\right]_{x} \\
& \mathrm{M}^{\prime}\left(\mathrm{O}^{\mathrm{t}} \mathrm{Bu}\right)_{3} \mathrm{M}-\mathrm{M}^{\mathrm{T}}(\mathrm{CO})_{n} \frac{h^{v}}{\mathrm{THF}} \mathrm{M}^{\prime}\left(\mathrm{O}^{\mathrm{t}} \mathrm{Bu}\right)_{3} \mathrm{M}^{\mathrm{T}}(\mathrm{CO})_{n-1} \cdot \mathrm{THF}+\mathrm{CO} \stackrel{\Delta}{\longrightarrow} \\
& \quad 1 / x\left[-\mathrm{M}^{\prime}\left(\mathrm{O}^{\mathrm{t}} \mathrm{Bu}\right)_{3} \mathrm{M}^{\mathrm{T}}(\mathrm{CO})_{n-1}-\right]_{x}+\mathrm{THF} \\
& (\mathrm{CO})_{n} \mathrm{M}^{\mathrm{T}}-\mathrm{M}^{\prime}\left(\mathrm{O}^{\mathrm{t}} \mathrm{Bu}\right)_{3} \mathrm{M}-\mathrm{M}^{\mathrm{T}}(\mathrm{CO})_{n}+\mathrm{M}^{\prime}\left(\mathrm{O}^{\mathrm{t} B u}\right)_{3} \mathrm{M} \rightarrow \\
& (\mathrm{CO})_{n} \mathrm{M}^{\mathrm{T}}-\mathrm{M}^{\prime}\left(\mathrm{O}^{\mathrm{t}} \mathrm{Bu}\right)_{3} \mathrm{M}-\mathrm{M}^{\mathrm{T}}(\mathrm{CO})_{n-1} \mathrm{M}^{\prime}(\mathrm{OtBu})_{3} \mathrm{M} \stackrel{-\mathrm{CO}}{\longrightarrow} \text { etc. }
\end{aligned}
$$

Unfortunately, none of these reactions give the desired products; instead, equilibria of mono- $\left[(\mathrm{CO})_{n} \mathbf{M}^{\mathrm{T}}-\mathrm{M}(\mathrm{OtBu})_{3} \mathrm{M}^{\prime}\right]$ and dicoordinated alkoxides $\left[(\mathrm{CO})_{n} \mathbf{M}^{\mathbf{T}}-\right.$ $\left.\mathrm{M}^{\prime}\left(\mathrm{O}^{t} \mathrm{Bu}\right)_{3} \mathrm{M}-\mathrm{M}^{\mathrm{T}}(\mathrm{CO})_{n}\right]$ are obtained. If the temperatures are raised too high rapid degradation of the starting materials is observed.

Switching from $\mathrm{M}\left(\mathrm{O}^{t} \mathrm{Bu}\right)_{3} \mathrm{M}^{\prime}$ to the system $\mathrm{M}^{\prime}\left(\mathrm{O}^{t} \mathrm{Bu}\right)_{3} \mathrm{M}\left(\mathrm{O}^{t} \mathrm{Bu}\right)_{3} \mathrm{M}^{\prime}$ does not improve the results (with respect to the formation of oligomers). Nevertheless, when $\mathrm{Sn}\left(\mathrm{O}^{t} \mathrm{Bu}\right)_{3} \mathrm{Sr}\left(\mathrm{O}^{t} \mathrm{Bu}\right)_{3} \mathrm{Sn}$ reacts with norbornadiene chromium tetracarbonyl, an insoluble yellow powder is obtained which shows one IR absorption in the $\mathrm{CO}$ region $\left(1880 \mathrm{~cm}^{-1}\right)$. From the analytical data this product seems to be a mixture of $\mathrm{Sn}\left(\mathrm{O}^{t} \mathrm{Bu}\right)_{3} \mathrm{Sr}\left(\mathrm{O}^{t} \mathrm{Bu}\right)_{3} \mathrm{Sn}-\mathrm{Cr}(\mathrm{CO})_{4}-\mathrm{Sn}\left(\mathrm{O}^{t} \mathrm{Bu}\right)_{3} \mathrm{Sr}\left(\mathrm{O}^{\mathrm{t}} \mathrm{Bu}\right)_{3} \mathrm{Sn}$ and $\left[\mathrm{Sn}\left(\mathrm{O}^{t} \mathrm{Bu}\right)_{3} \mathrm{Sr}\right.$ $\left.\left(\mathrm{O}^{\mathrm{t}} \mathrm{Bu}\right)_{3} \mathrm{Sn}-\mathrm{Cr}(\mathrm{CO})_{4}\right]_{x}$. Unfortunately, we have no yet been able to separate these compounds.

Very recently we have been successful in the synthesis of the first oligomeric metal carbonyl-metal alkoxide systems [eqn. (17)].

$$
\begin{aligned}
& (n+1) \mathrm{M}^{\prime}\left(\mathrm{O}^{t} \mathrm{Bu}\right)_{3} \mathrm{In}-\mathrm{Mo}(\mathrm{CO})_{5} \underset{-n \mathrm{CO}}{\stackrel{\Delta}{\longrightarrow}} \\
& {\left[\mathrm{M}^{\prime}\left(\mathrm{O}^{\mathrm{t}} \mathrm{Bu}\right)_{3} \mathrm{In}-\mathrm{Mo}(\mathrm{CO})_{4}\right]_{n}-\mathrm{M}^{\prime}\left(\mathrm{O}^{\mathrm{t}} \mathrm{Bu}\right)_{3} \mathrm{In}-\mathrm{Mo}(\mathrm{CO})_{5}} \\
& \mathrm{M}^{\prime}=\mathrm{Ge}, \mathrm{Sn}
\end{aligned}
$$

The two starting materials GIM and SIM behave slightly differently. Upon thermolysis of GIM in refluxing xylene the product precipitates, whereas the thermolysis of SIM works best in the melt at about $135^{\circ} \mathrm{C}$. An insoluble product is again obtained which deposits from the melt.

The oligomer obtained from SIM has been extensively studied. Its IR spectrum contains three major bands in the carbonyl stretching region [2012 $\left(A_{1}^{1 \mathrm{~b}}\right), 1940$ 
$\left.\left(A_{1}^{12}\right), 1891\left(B_{1}\right)\right]$. All bands are shifted to lower wavenumbers compared with SIM (Table 3), which is an indication of a second coordination of a main group metal at the molybdenum centres. Further the difference in the high-frequency $A_{1}$ band between SIM and its oligomer $\left(\Delta v 49 \mathrm{~cm}^{-1}\right)$ is typical for the transition from mono- to disubstitution, as may be concluded from the pairs of complexes $(\mathrm{CO})_{5} \mathrm{Mo}\left[\mathrm{MeP}(\mathrm{OMe})_{2}\right]$ versus $(\mathrm{CO})_{4} \mathrm{Mo}\left[\mathrm{MeP}(\mathrm{OMe})_{2}\right]_{2} \quad\left(\Delta v=47 \mathrm{~cm}^{-1}\right)$ [38], $(\mathrm{CO})_{5} \mathrm{Mo}\left(\mathrm{AsPh}_{3}\right)$ versus $(\mathrm{CO})_{4} \mathrm{Mo}\left(\mathrm{AsPh}_{3}\right)_{2} \quad\left(\Delta v=51 \mathrm{~cm}^{-1}\right) \quad$ [39] and $(\mathrm{CO})_{5} \mathrm{Mo}\left(\mathrm{AsEt}_{3}\right)$ versus $(\mathrm{CO})_{4} \mathrm{Mo}\left(\mathrm{AsEt}_{3}\right)_{2}\left(\Delta v=55 \mathrm{~cm}^{-1}\right)$ [40]. In addition, the $n \mathrm{~s}^{2}$ element substituents are (at least to a significant extent) in a cis arrangement as determined from the IR absorption patterns. A partial trans arrangement cannot be excluded, however, since the single absorption band of the trans isomer (local symmetry $D_{4 \mathrm{~h}}$ ) would be in the same region as the strong $B_{1}$ band of the cis isomer (local symmetry $C_{2 \mathrm{v}}$ ). There is a tiny absorption shoulder at $2055 \mathrm{~cm}^{-1}$, which we attribute to the trans-CO stretch in an $\mathrm{Mo}(\mathrm{CO})_{5}$ group (compare MISM or MISC). This may be indicative of a terminal group and therefore it seems reasonable to assume that the product is not a cyclic oligomer but a chain oligomer. From the intensity ratio of the $A_{1}$ band at $2012 \mathrm{~cm}^{-1}$ and the shoulder at $2055 \mathrm{~cm}^{-1}$, a rough estimation may be made of the number of units $n$ in $\left[\mathrm{Sn}\left(\mathrm{O}^{t} \mathrm{Bu}\right)_{3} \mathrm{In}-\mathrm{Mo}(\mathrm{CO})_{4}^{-}\right]_{n}$ $\mathrm{Sn}\left(\mathrm{O}^{\mathrm{t}} \mathrm{Bu}\right)_{3} \mathrm{In}-\mathrm{Mo}(\mathrm{CO})_{5}$, which should be about 10 .

In addition to the analytical data, which are in accord with the oligomer, the quantitative gas evolution has been studied by gas volumetry and by mass spectrometry and unambiguously shows the only gas being evolved during the reaction to be carbon monoxide. The volumetry results in $85-90 \% \mathrm{CO}$ evolution starting with the pure compound SIM. Taking this number into account, a ratio $\mathrm{Mo}(\mathrm{CO})_{4}$ to $\mathrm{Mo}(\mathrm{CO})_{5}$ of $9: 1$ in the product is calculated, which is in accord with the result from IR spectroscopy. Thus the chain length should be $n=9$ or 10 in $\left[\mathrm{Sn}\left(\mathrm{O}^{\mathrm{t}} \mathrm{Bu}\right)_{3} \mathrm{In}-\mathrm{Mo}(\mathrm{CO})_{4}\right]_{n} \mathrm{Sn}\left(\mathrm{O}^{\mathrm{t}} \mathrm{Bu}\right)_{3} \mathrm{In}-\mathrm{Mo}(\mathrm{CO})_{5}$.

We have not yet determined if the product is uniform or if there is a mixture of several different oligomeric species. The immediate precipitation from the melt indicates that the degree of oligomerization does not vary very much. Unfortunately, we were not able to record a ${ }^{119} \mathrm{Sn}$ MAS (magic angle spinning) NMR spectrum of the solid, as the molybdenum isotope ${ }^{97} \mathrm{Mo}$ has a high quadrupole moment. On the other hand, this even seems to confirm that $\mathrm{Sn}$ is indeed bound to Mo. The ${ }^{13} \mathrm{C} \mathrm{CP}$ (cross-polarization) MAS NMR spectrum is fairly simple with resonances at 34.7, $76.8,205$ and $209 \mathrm{ppm}$ and is different from that of the starting compound SIM (35.8, 70.4, 200 and $203 \mathrm{ppm}$ in benzene solution). Interestingly, the resonances for the $\mathrm{CO}$ groups are shifted to higher $\delta$-values, which is again indicative of a second substitution at molybdenum [41]. The signals of the tert-butyl groups are sharp (indicative of a uniform product), whereas the resonance signals for the carbonyls are broad and overlapping. Unfortunately, they cannot be resolved in order to distinguish the $\mathrm{CO}$ end-group [for experimental reasons (signal-to-noise ratio and relaxation time), the ${ }^{13} \mathrm{C}$ CP-MAS NMR techniques give better results than the 
MAS experiment, especially in the $\mathrm{CO}$ resonance region]. The oligomers obtained from eqn. (17) are pale yellow and darken on contact with air. So far no physical measurements on cooperative effects along these one-dimensional, "percolated" metal rows have been performed.

\section{ACKNOWLEDGEMENTS}

We thank the Fonds der Chemischen Industrie and the Deutsche Forschungsgemeinschaft for financial support.

\section{REFERENCES}

1 M. Petz, Chem. Rev., 86 (1986) 1019.

2 M. Veith, Angew. Chem., 99 (1987) 1; Angew. Chem., Int. Ed. Engl., 26 (1987) 1.

3 M. Veith, Phosphorus Sulfur Silica, 41 (1989) 195.

4 M. Veith, Chem. Rev., 90 (1990) 3.

5 Janus, Roman god with two faces; January is the month pointing in two opposite directions: one to the old year, the other to the new year; in the "Janus-Head" molecules the metallic elements are situated on opposite faces of the $\mathrm{O}_{3}$ triangle.

6 R.C. Mehrotra, Adv. Inorg. Chem. Radiochem., 26 (1983) 269.

7 D.C. Bradley, Chem. Rev., 89 (1989) 1317.

8 K.C. Caulton and L.G. Hubert-Pfalzgraf, Chem. Rev., 90 (1990) 969.

9 M. Veith, J. Hans, L. Stahl, P. May, V. Huch and A. Sebald, Z. Naturforsch., Teil B, 46 (1991) 403.

10 M. Veith and R. Rösler, Angew. Chem., 94 (1982) 867; Angew. Chem., Int. Ed. Engl., 21 (1982) 858.

11 M. Veith and K. Kunze, Angew. Chem., 103 (1991) 92; Angew. Chem., Int. Ed. Engl., 30 (1991) 95.

12 M. Veith and R. Rösler, Z. Naturforsch., Teil B, 41 (1986) 1071.

13 M. Veith, D. Käfer, J. Koch, P. May, L. Stahl and V. Huch, Chem. Ber., 125 (1992) 1033.

14 W. Strohmeier and K. Gerlach, Chem. Ber., 94 (1961) 398.

15 W. Strohmeier, Angew. Chem., 76 (1964) 873; Angew. Chem., Int. Ed. Engl., 3 (1964) 730.

16 M. Grenz and W.W. du Mont, J. Organomet. Chem., 241 (1983) C5.

17 M. Grenz, E. Hahn, W.W. du Mont and J. Pickardt, Angew. Chem., 96 (1984) 69; Angew. Chem., Int. Ed. Engl., 23 (1984) 61.

18 P.B. Hitchcock, M.F. Lappert, S.A. Thomas and A.J. Thorne, J. Organomet. Chem., 315 (1986) 27.

19 M.A. Bennett, L. Pratt and G. Wilkinson, J. Chem. Soc., (1961) 2037.

20 E.W. Abel and F.G.A. Stone, Q. Rev. Chem. Soc., 24 (1970) 498.

21 F.A. Cotton, Prog. Inorg. Chem., 21 (1976) 1.

22 A. Vincent, Molecular Symmetry and Group Theory, Wiley, Chichester, 1977.

23 J. Weidlein, U. Müller and K. Dehnicke, Schwingungsspektroskopie, Georg Thieme, Stuttgart, 1982.

24 M. Veith, H. Lange, K. Bräuer and R. Bachmann, J. Organomet. Chem., 216 (1981) 377.

25 J.D. Cotton, P.J. Davidson and M.F. Lappert, J. Chem. Soc., Dalton Trans., (1976) 2275.

26 J.D. Cotton, P.J. Davidson, D.E. Goldberg, M.F. Lappert and K.M. Thomas, J. Chem. Soc., Chem. Commun., (1974) 893. 
27 D.F. Shriver, P.W. Atkins and C.H. Langford, Inorganic Chemistry, Oxford University Press, Oxford, 1990.

28 Further details of the crystal structure investigations may be obtained from the Fachinformationszentrum Karlsrube, Gesellschaft für Wissenschaftliche Information $\mathrm{mbH}$, D-76344 Eggenstein-Leopoldshafen, Germany, on quoting the depository number CSD 58225 , the names of the authors and the journal citation.

29 M. Veith and J. Hans, Angew. Chem., 103 (1991) 845; Angew. Chem., Int. Ed. Engl., 30 (1991) 878.

30 F.A. Cotton and C.S. Kraihanzl, J. Am. Chem. Soc., 84 (1962) 4432.

31 J.D. Atwood and T.L. Brown, J. Am. Chem. Soc., 98 (1976) 3160.

32 J.K. Burdett, Inorg. Chem., 14 (1975) 375.

33 D.L. Lichtenberger and T.L. Brown, J. Am. Chem. Soc., 100 (1978) 366.

34 J. Dalton, I. Paul, J.G. Smith and F.G.A. Stone, J. Chem. Soc. A, (1968) 1195.

35 R.J. Mawby and C. White, J. Chem. Soc., Chem. Commun., (1968) 312.

$36 \mathrm{M}$. Veith and $\mathrm{K}$. Kunze, results to be published.

37 H. Preut and H.J. Haupt, Acta Crystallogr., Sect. B, 35 (1979) 2191.

38 G. Bouquet and M. Bigorgne, Bull. Soc. Chim. Fr., (1962) 433.

39 C.E. Jones and K.J. Coskran, Inorg. Chem., 10 (1971) 55.

40 E.O. Fischer and R.J.J. Schneider, Chem. Ber., 103 (1970) 3684.

41 L.J. Todd and J.R. Wilkinson, J. Organomet. Chem., 77 (1974) 1. 